QUARTERLY OF APPLIED MATHEMATICS

VOLUME LXIII, NUMBER 1

MARCH 2005, PAGES 88-116

S $0033-569 X(05) 00953-9$

Article electronically published on January 20, 2005

\title{
LOCAL EXISTENCE AND UNIQUENESS FOR QUASISTATIC FINITE PLASTICITY WITH GRAIN BOUNDARY RELAXATION
}

\author{
BY \\ PATRIZIO NEFF \\ AG6, Fachbereich Mathematik, Darmstadt University of Technology, Schlossgartenstrasse 7, 64289 \\ Darmstadt, Germany
}

\begin{abstract}
This paper is concerned with a phenomenological model of initially isotropic finite-strain multiplicative elasto-plasticity for polycrystals with grain boundary relaxation (Neff, Cont. Mech. Thermo., 2003). We prove a local in time existence and uniqueness result of the corresponding initial boundary value problem in the quasistatic rate-dependent case. Use is made of a generalized Korn first inequality (Neff, Proc. Roy. Soc. Edinb. A, 2002) taking into account the incompatibility of the plastic deformation $F_{p}$. This is a first result concerning classical solutions in geometrically exact nonlinear finite visco-plasticity for polycrystals. Global existence is not proved and cannot be expected due to the natural possibility of material degradation in time.
\end{abstract}

1. Notation. Let $\Omega \subset \mathbb{R}^{3}$ be a bounded domain with Lipschitz boundary $\partial \Omega$ and let $\Gamma$ be a smooth subset of $\partial \Omega$ with nonvanishing 2-dimensional Hausdorff measure. For two Banach spaces $X, Y$ we let $\operatorname{Lin}(X, Y)$ denote the vectorspace of all bounded linear mappings from $X$ to $Y$ and $\mathcal{P}(X)$ denotes the set of all subsets of $X$. For $a, b \in \mathbb{R}^{3}$ we let $\langle a, b\rangle_{\mathbb{R}^{3}}$ denote the scalar product on $\mathbb{R}^{3}$ with associated vector norm $\|a\|_{\mathbb{R}^{3}}^{2}=\langle a, a\rangle_{\mathbb{R}^{3}}$. We denote by $\mathbb{M}^{3 \times 3}$ the set of real $3 \times 3$ second order tensors, written with capital letters. The standard Euclidean scalar product on $\mathbb{M}^{3 \times 3}$ is given by $\langle X, Y\rangle_{\mathbb{M}^{3 \times 3}}=$ $\operatorname{tr}\left[X Y^{T}\right]$, and thus the Frobenius tensor norm is $\|X\|^{2}=\langle X, X\rangle_{\mathbb{M}^{3 \times 3}}$. In the following we omit the index $\mathbb{R}^{3}, \mathbb{M}^{3 \times 3}$. The identity tensor on $\mathbb{M}^{3 \times 3}$ will be denoted by $\mathbb{1}$, so that $\operatorname{tr}[X]=\langle X, \mathbb{1}\rangle$. We let Sym and PSym denote the symmetric and positive definite symmetric tensors, respectively. We adopt the usual abbreviations of Lie-group theory, i.e., $\mathrm{GL}(3, \mathbb{R}):=\left\{X \in \mathbb{M}^{3 \times 3} \mid \operatorname{det}[X] \neq 0\right\}$ is the general linear group, $\mathrm{SL}(3, \mathbb{R}):=$ $\{X \in \mathrm{GL}(3, \mathbb{R}) \mid \operatorname{det}[X]=1\}, \mathrm{O}(3):=\left\{X \in \mathrm{GL}(3, \mathbb{R}) \mid X^{T} X=\mathbb{1}\right\}, \quad \mathrm{SO}(3, \mathbb{R}):=$

Received May 13, 2004.

2000 Mathematics Subject Classification. Primary 74A35, 74C05, 74C10, 74C20, 74D10, 74E05, 74E10, 74E15, 74G30, 74G65, 74N15.

Key words and phrases. Plasticity, visco-plasticity, solid mechanics, elliptic systems, variational methods.

Current address: Department of Mathematics, University of Technology, Darmstadt.

E-mail address: neff@mathematik.tu-darmstadt.de

(C)2005 Brown University 
$\left\{X \in \mathrm{GL}(3, \mathbb{R}) \mid X^{T} X=\mathbb{1}, \operatorname{det}[X]=1\right\}$ with corresponding Lie algebras $\mathfrak{s o}(3):=\{X \in$ $\left.\mathbb{M}^{3 \times 3} \mid X^{T}=-X\right\}$ of skew symmetric tensors and $\mathfrak{s l}(3):=\left\{X \in \mathbb{M}^{3 \times 3} \mid \operatorname{tr}[X]=0\right\}$ of traceless tensors. With Adj $X$ we denote the tensor of transposed cofactors $\operatorname{Cof}(\mathrm{X})$ such that $\operatorname{Adj} X=\operatorname{det}[X] X^{-1}=\operatorname{Cof}(\mathrm{X})^{\mathrm{T}}$ if $X \in \mathrm{GL}(3, \mathbb{R})$. We set $\operatorname{sym}(X)=\frac{1}{2}\left(X^{T}+X\right)$ and $\operatorname{skew}(X)=\frac{1}{2}\left(X-X^{T}\right)$ such that $X=\operatorname{sym}(X)+\operatorname{skew}(X)$. For $X \in \mathbb{M}^{3 \times 3}$ we set for the deviatoric part $\operatorname{dev} X=X-\frac{1}{3} \operatorname{tr}[X] \mathbb{1} \in \mathfrak{s l}(3)$ and for vectors $\xi, \eta \in \mathbb{R}^{n}$ we have the tensor product $(\xi \otimes \eta)_{i j}=\xi_{i} \eta_{j}$. We write the polar decomposition in the form $F=R U=\operatorname{polar}(F) U$ with $R=\operatorname{polar}(F)$ the orthogonal part of $F$. In general we work in the context of nonlinear, finite elasticity. For the total deformation $\varphi \in C^{1}\left(\bar{\Omega}, \mathbb{R}^{3}\right)$ we have the deformation gradient $F=\nabla \varphi \in C\left(\bar{\Omega}, \mathbb{M}^{3 \times 3}\right)$. Furthermore, $S_{1}(F)$ and $S_{2}(F)$ denote the first and second Piola Kirchhoff stress tensors, respectively. Total time derivatives are written $\frac{\mathrm{d}}{\mathrm{dt}} X(t)=\dot{X}$. The first and second differentials of a scalarvalued function $W(F)$ are written $D_{F} W(F) \cdot H$ and $D_{F}^{2} W(F) \cdot(H, H)$, respectively. We set $C=F^{T} F, C_{p}=F_{p}^{T} F_{p}, C_{e}=F_{e}^{T} F_{e}, E=\frac{1}{2}(C-\mathbb{1}), E_{p}=\frac{1}{2}\left(C_{p}-\mathbb{1}\right), E_{e}=\frac{1}{2}\left(C_{e}-\mathbb{1}\right)$. We employ the standard notation of Sobolev spaces, i.e., $L^{2}(\Omega), H^{1,2}(\Omega), H_{\circ}^{1,2}(\Omega)$, which we use indifferently for scalar-valued functions as well as for vector-valued and tensorvalued functions. Moreover, we set $\|X\|_{\infty}=\sup _{x \in \Omega}\|X(x)\|$. For $X \in C^{1}\left(\bar{\Omega}, \mathbb{M}^{3 \times 3}\right)$ we define $\operatorname{Curl} X(x)$ as the operation curl applied rowwise. We define $H_{\circ}^{1,2}(\Omega, \Gamma):=\{\phi \in$ $\left.H^{1,2}(\Omega) \mid \phi_{\left.\right|_{\Gamma}}=0\right\}$, where $\phi_{\left.\right|_{\Gamma}}=0$ is to be understood in the sense of traces and by $C_{0}^{\infty}(\Omega)$ we denote infinitely differentiable functions with compact support in $\Omega$. We use capital letters to denote possibly large positive constants, e.g., $C^{+}, K$, and lowercase letters to denote possibly small positive constants, e.g,. $c^{+}, d^{+}$. The smallest eigenvalue of a positive definite symmetric tensor $P$ is abbreviated by $\lambda_{\min }(P)$. The Landau symbols $o(h), O(h)$ are employed. Finally, w.r.t. abbreviates "with respect to".

\section{Introduction.}

2.1. Approaches in plasticity theory. Plasticity theory, as it is understood today, covers a large field of study which involves difficult phenomena in the inelastic behaviour of solids along with difficult analytical problems related to the nonlinearity of the employed mathematical models.

"As is well known, the existing formulations of a general theory of elastic-plastic material in the presence of finite deformations are somewhat controversial and there remains strong disagreement on a number of important issues between several schools of plasticity" [51, p. 317]. More than fourteen years after these comments by Naghdi have been made, the issues are still far from being settled. Without being exhaustive, these fundamental disagreements concern the following topics (references only to illustrate the diverging approaches):

(1) flow theory versus total deformation theory of plasticity (Hencky model),

(2) stress- or strain-based approach [51,

(3) additive decomposition $E=E_{e}+E_{p}$ of total strain [24, 12, 51] into symmetric elastic and plastic parts versus multiplicative decomposition $F=F_{e} F_{p}$ of deformation [32, 38, 41 into elastic and plastic parts,

(4) plastic strain $E_{p} \in \operatorname{Sym}(3)$ as primitive phenomenological variable versus the deduced quantity $E_{p}=\frac{1}{2}\left(F_{p}^{T} F_{p}-\mathbb{1}\right)$, 
(5) invariance and covariance requirements on the plastic strain $E_{p}[12$ versus the concept of an elastic isomorphism and the natural reference state [5, 3],

(6) interpretation of the multiplicative decomposition in terms of an intermediate, locally unloaded, stress-free configuration [38] versus an isoclinic configuration [5, 41],

(7) Eulerian approach (hypo-plastic formulation, question of objective stress-rates) [46, 9] versus Lagrangian approach (hyper-elastoplastic, circumventing discussion of stress-rates) $26,3,62$,

(8) rate-independent formulations (instantaneous complete energy relaxation) [11] versus rate-dependent formulations (viscosity, creep, relaxation, fatigue) [28,

(9) associative (metals) or nonassociative flow rules (geomaterials) 74]; possibility of variational time-incremental updates for associative formulations [62, 60, 11] versus the more traditional coupled evolution problem,

(10) yield surface approach (von Mises, Tresca) with elastic region versus unified constitutive equations [8, 66] without yield limit,

(11) formulation of anisotropic behaviour; significance of plastic spin [19,

(12) inclusion of thermal effects and kinematical hardening.

These points clearly illustrate the nonexistence of some encompassing theory of largestrain plasticity.

Practically all developments on the subject related to infinitesimal deformations of elastic-plastic material have adopted a flow theory, stress-based approach and have utilized yield surfaces and associated loading criteria. One can conclude that a satisfactory level of agreement can be reached as far as infinitesimal elasto-plasticity is concerned.

The difference in the formulations appears prominently only when finite deformations are considered.

While it is not possible to mathematically resolve the apparent disagreement on the formulation, our interest is on those models, which are based on the multiplicative decomposition, along with a stress-based formulation and which include from the outset rate-dependent effects. The rate-independent behaviour is included as a certain purely mathematical limiting response. Attention is restricted to isothermal, isotropic formulations for simplicity without hardening.

We briefly recapitulate basic points of the multiplicative decomposition and introduce the nonlinear initial boundary value problems to be solved. Certain intrinsic problems of these formulations are hinted at. In order to sidestep these problems, we introduce a new, geometrically exact model with grain boundary relaxation and include a brief discussion of invariance requirements. A local existence and uniqueness result for this model is stated.

Thereafter, we explain the basic mathematical ideas with which to show this wellposedness result. The remaining part is devoted to the technical details of the proof.

In the appendix we introduce the generalized Korn first inequality as well as elliptic regularity results needed for the proof which makes the presentation sufficiently selfcontained.

2.2. Recapitulation of finite multiplicative plasticity. In the nonlinear theory of elastovisco-plasticity at large deformation gradients it is often assumed that the deformation 
gradient $F=\nabla \varphi$ splits multiplicatively] into an elastic and plastic part [38, 41]

$$
\nabla \varphi(x)=F(x)=F_{e}(x) \cdot F_{p}(x), \quad F_{e}, F_{p} \in \mathrm{GL}^{+}(3, \mathbb{R}),
$$

where the invertible tensors $F_{e}, F_{p}$ are explicitly understood to be incompatible configurations, i.e., $F_{e}, F_{p} \neq \nabla \Psi$ for any $\Psi: \Omega \subset \mathbb{R}^{3} \mapsto \mathbb{R}^{3}$. Thus $F_{p}$ introduces in a natural way a non-Riemannian manifold structure [33]. While formally this decomposition is unique only up to an invertible matrix $G \in \mathrm{GL}(3, \mathbb{R})$, since

$$
\nabla \varphi(x)=F(x)=F_{e}(x) \cdot F_{p}(x)=F_{e}(x) G(x) G(x)^{-1} F_{p}(x)=\tilde{F}_{e}(x) \cdot \tilde{F}_{p}(x),
$$

we consider as "physically equivalent" decompositions only those obtained by a global rigid rotation $F_{e}(x) Q$ with $Q \in \mathrm{SO}(3)$. In addition one sometimes assumes isochoric plastic deformations only, i.e., $\operatorname{det}\left[F_{p}(x)\right]=1$, notably in metal-plasticity. This multiplicative split, which has gained more or less permanent status in the literature, can be micro-mechanically motivated by the kinematics of single crystals where dislocations move along fixed slip systems through the crystal lattice. The sources for the incompatibility are those dislocations which did not completely transverse the crystal and consequently give rise to an inhomogeneous plastic deformation. Therefore, in the case of single crystal plasticity it is reasonable to introduce the deviation of the plastic intermediate configuration $F_{p}$ from compatibility as a kind of plastic dislocation density. This deviation should be related somehow to the quantity Curl $F_{p}$ and indeed in the contribution [56] we see the important role which is played by Curl $F_{p}$ for coerciveness inequalities related to the existence theory of models in this area.

The constitutive assumption (2.1) is incorporated into the balance of the linear momentum governing the elastic response of the material and supplemented by flow rules in the form of ordinary differential equations or differential inclusions determining the evolution of the plastic part. In the general case the equations of elasto-plasticity take the form

$$
\begin{gathered}
\varrho \varphi_{t t}=\operatorname{Div} D_{F}\left[W\left(\nabla \varphi(t, x) \cdot F_{p}^{-1}(t, x)\right)\right]+f(x), \quad x \in \Omega, \\
\frac{\mathrm{d}}{\mathrm{dt}}\left[F_{p}^{-1}\right](t, x) \in F_{p}^{-1}(t, x) \cdot \mathfrak{f}\left(\nabla \varphi(t, x), F_{p}^{-1}(t, x)\right),
\end{gathered}
$$

supplemented with initial and boundary conditions. Here $W$ is the elastic-free energy density defined on the elastic part $F_{e}, \varrho>0$ is the mass density, $f$ is the body force and $\mathfrak{f}: D(\mathfrak{f}) \mapsto \mathcal{P}\left(\mathbb{M}^{3 \times 3}\right)$ is the possibly set-valued monotone flow function with domain of definition $D(\mathfrak{f})$. In this generality ( $(\underline{\mathrm{P} 0})$ comprises single and polycrystal plasticity theory and the rate-dependent as well as the rate-independent (set-valued) case of associated or nonassociated plasticity.

We refer the reader to [7, 32, 35, 36, 43, 71, 13] for more on the subject of dislocations and incompatibilities and to 61 for an account of the occurrence of microstructure. A recent summary presentation of the theory for single crystals can be found in [25]. For applications of the general theory of polycrystalline materials in the engineering field look, e.g., at [47, 70, 68, 69, 17, 18]. An introduction to the theory of materials in general

\footnotetext{
${ }^{1}$ While we continue to use the terms multiplicative decomposition and intermediate configuration, it is rather an elastic isomorphism in the sense of [3]. Some authors use $P$ instead of $F_{p}^{-1}$ 11.
} 
and inelastic deformations can be found in 29, 5, 39]. Abstract mathematical treatments concerning the modelling of elasto-plasticity may be found in [67, 40].

The multiplicative split (2.1) can also be seen as a generalization to finite deformations of the well known additive decomposition

$$
\frac{1}{2}\left(\nabla u+\nabla u^{T}\right)=\varepsilon(\nabla u(x))=\varepsilon_{e}(x)+\varepsilon_{p}(x),
$$

where we have set $F=\mathbb{1}+\nabla u$ with $u$ the displacement vector and where subsequently $\varepsilon(\nabla u(x))$ denotes the infinitesimally strain tensor. This additive decomposition is appropriate only for infinitesimally small values of $\|\nabla u\|$. There is a rich mathematical literature successfully treating plasticity models based on (2.3) (cf.(2.17)) of the type

$$
\varrho u_{t t}=\operatorname{Div} \mathbb{D}_{\operatorname{lin}} \cdot\left(\varepsilon(\nabla u)-\varepsilon_{p}\right)+f, \quad \dot{\varepsilon}_{p} \in \mathfrak{f}\left(-\mathbb{D}_{\operatorname{lin}} \cdot\left(\varepsilon(\nabla u)-\varepsilon_{p}\right)\right),
$$

with $\varepsilon_{p} \in \mathfrak{s l}(3, \mathbb{R})$. See, e.g., [1, 27, 30, 14, 15] and references therein. In [1] the flow rule in the form of (2.4) is called of pre-monotone type if $\langle\mathfrak{f}(\Sigma), \Sigma\rangle \geq 0$.

Although there is an abundance of applications and numerical simulations involving finite strains for single or polycrystals, up to the present a rigorous mathematical treatment (and a convergence proof for discretizations) of the general case ([P0) is missing. This is mainly due to two facts: the finite elasticity part involved in (P0) itself is difficult to analyze and the flow rule is highly nonlinear with additional peculiar properties in the rate-independent (set-valued) limit case. A promising approach towards a mathematical analysis for the quasistatic rate-independent case of associated single crystal plasticity which is based on a time discrete incremental variational formulation can be found in $[10,48,60$.

In metal-plasticity, as for most crystalline materials, one observes that the shape of the elastically deformed crystals remains nearly unchanged [39, 3, 65]. In the context of multiplicative elasto-plasticity this translates into $\left\|\frac{F_{e}^{T} F_{e}}{\operatorname{det}\left[F_{e}\right]^{2 / 3}}-\mathbb{1}\right\|$ pointwise small where we assume that $F_{e}$ is locally invertible. In most applications, however, elastic volume changes are also negligible, leaving us with $\left\|F_{e}^{T} F_{e}-\mathbb{1}\right\|$ small. In addition one can assume isotropic behaviour for a polycrystal, since the different orientations of the crystal grains average out.

Therefore, let us look at finite hyperelasticity for small strains. We assume the existence of a free elastic energy $\hat{W}=\hat{W}(F)=\hat{W}(\nabla \varphi)$. This constitutive relation is subjected to the material frame indifference, i.e., it must remain invariant under superimposed rigid body motions. Together with the isotropy of $\hat{W}$, homogeneity and the requirement that $D \hat{W}(\mathbb{1})=0$, i.e., that the reference configuration be stress free, it can be shown [16, p. 156] that $\hat{W}=\hat{W}(F)$ and

$$
\hat{W}(F)=\mu\|U-\mathbb{1}\|^{2}+\frac{\lambda}{2} \operatorname{tr}[U-\mathbb{1}]^{2}+o\left(\|U-\mathbb{1}\|^{2}\right)=\mu\|E\|^{2}+\frac{\lambda}{2} \operatorname{tr}[E]^{2}+o\left(\|E\|^{2}\right)
$$

holds near a natural state where $E=\frac{1}{2}\left(F^{T} F-\mathbb{1}\right)$ denotes the Green-Lagrange strain tensor and $U-\mathbb{1}$ denotes the Biot strain tensor. The Lamé constants $\mu, \lambda$ of the polycrystalline material under consideration are assumed to be nonnegative throughout with 
$\mu>0$. This energy can be used as a starting point for the definition of a suitable elasticplastic energy in as much as one makes the ansatz $\hat{W}=\hat{W}\left(F_{e}\right)$ where one has simply substituted the elastic part of the deformation gradient $F_{e}$ instead of $F$.

In view of the small elastic strain assumption, it is reasonable to ignore the dependence of the elastic energy $\hat{W}\left(F_{e}\right)$ on the higher order term $o\left(\left\|F_{e}^{T} F_{e}-\mathbb{1}\right\|^{2}\right)$ and one is left with the nonlinear Saint-Venant Kirchhoff energy $W\left(F_{e}\right)=\frac{\mu}{4}\left\|F_{e}^{T} F_{e}-\mathbb{1}\right\|^{2}+$ $\frac{\lambda}{8} \operatorname{tr}\left[F_{e}^{T} F_{e}-\mathbb{1}\right]^{2}$.

In the quasistatic setting we arrive at the following system of coupled partial differential and evolution equations for the deformation $\varphi:[0, T] \times \bar{\Omega} \mapsto \mathbb{R}^{3}$ and the plastic deformation $F_{p}:[0, T] \times \bar{\Omega} \mapsto \mathrm{GL}(3, \mathbb{R})$ :

$$
\begin{aligned}
0 & =\operatorname{Div} D_{F}\left[W\left(\nabla \varphi(t, x) \cdot F_{p}^{-1}(t, x)\right)\right]+f(x), \quad x \in \Omega, \quad \varphi_{\left.\right|_{\Gamma}}=g(t, x), \quad x \in \Gamma, \\
W\left(F_{e}\right) & =\frac{\mu}{4}\left\|F_{e}^{T} F_{e}-\mathbb{1}\right\|^{2}+\frac{\lambda}{8} \operatorname{tr}\left[F_{e}^{T} F_{e}-\mathbb{1}\right]^{2}, \\
\frac{\mathrm{d}}{\mathrm{dt}} & {\left[F_{p}^{-1}\right](t, x) \in F_{p}^{-1}(t, x) \cdot \mathfrak{f}\left(\nabla \varphi(t, x), F_{p}^{-1}(t, x)\right), \quad F_{p}^{-1}(0, x)=F_{p_{0}}^{-1} . }
\end{aligned}
$$

Here and subsequently $g(t, x)$ represents the time dependent inhomogeneous Dirichlet boundary data and $F_{p_{0}}^{-1}$ the initial condition for the plastic evolution.

Due to the still strong nonlinearity of (P1) it is not known whether the problem as such is well-posed, although the energy is quadratic in the elastic Green strains $E_{e}$ (physically linear). The reason for that is that the variational problem based on minimizing $F \mapsto$ $\left\|F_{e}^{T} F_{e}-\mathbb{1}\right\|^{2}$ at frozen $F_{p}$ may lead to microstructure since it is well known that the energy is not quasiconvex [64] and not even elliptic [56] in the compression range. The microstructure already inherent in the purely elastic formulation, however, should rather be seen as a (nonphysical) modelling artifact coming from the quadratic ansatz in $E_{e}$ since, e.g., when taking a corresponding compressible isotropic polyconvex Neo-Hooke energy $W\left(F_{e}\right)=\frac{\mu}{r}\left\|F_{e}\right\|^{r}+\frac{\lambda}{4} \operatorname{det}\left[F_{e}\right]^{2}-\frac{2 \mu+\lambda}{2} \ln \operatorname{det}\left[F_{e}\right]$, elastic microstructure cannot occur and the elastic solution is easily found in some Sobolev space if $F_{p} \in L^{\infty}(\Omega, \operatorname{GL}(3, \mathbb{R}))$. This and the underlying modelling ideas pertaining to the single crystal case suggest that $F_{p} \in L^{\infty}\left(\Omega, \mathrm{GL}^{+}(3, \mathbb{R})\right)$ is the minimal regularity we should impose in ( $\left.\mathrm{P} 0\right)$. The Neo-Hookean ansatz, however, falls short of taking into account small elastic strains. The above microstructure should therefore not be confused with the experimentally observed microstructure modelled with multi-well potentials in the theory of elastic crystals in connection with martensitic phase transformation [6].

In this contribution we study a novel model in order to give a partial answer to the question of well posedness of $(\mathrm{P0})$ in the rate-dependent quasistatic case. The main idea from a mathematical viewpoint is that the problem coming from finite elasticity can be completely circumvented in the (physically mostly relevant) case of small elastic strains without resorting either to the additive decomposition (2.3) or to (P1). To this end a new model appropriate for small elastic strains will be introduced whose first Piola-Kirchhoff stresses derive from a quadratic potential.

2.3. The model with grain boundary relaxation. One way to sidestep the above mentioned (apparently nonphysical purely mathematical) problems has been given by Neff [53, 57] where a modified model has been introduced. This model has as starting point 
(P1) but consequently incorporates the extra bit information of small elastic strains a priori. Let us recapitulate the main ingredients of the model for the presentation to be sufficiently self-contained.

In the three-dimensional case it is easily seen that small elastic strains, i.e., $\left\|F_{e}^{T} F_{e}-\mathbb{1}\right\|$ pointwise small for orientation preserving $F_{e} \in \mathrm{GL}^{+}(3, \mathbb{R})$, imply that $F_{e}$ is approximately a rotation $R_{e} \in \mathrm{SO}(3)$ (almost elastic rigidity). If we assume that $R_{e}$ is known, all quantities can be 'linearized' with respect to the local rigid configuration $R_{e}=\operatorname{polar}\left(F_{e}\right)$, the best approximation in terms of rotations to $F_{e}$. This is a nonlinear constraint. It is further possible to relax this static constraint into an evolution equation which describes internal relaxation such that a new visco-elastic rotation $\bar{R}_{e}$ is determined which coincides approximately with $\operatorname{polar}\left(F_{e}\right)$ whenever $F_{e}$ is approximately a rotation. The static constraint $R_{e}=\operatorname{polar}\left(F_{e}\right)$ is a global attractor of the evolution equation for $\bar{R}_{e}$. These modifications significantly change the mathematical structure without losing the main ingredients of finite multiplicative visco-plasticity, notably observer-invariance and invariance with respect to superposed rotations of the so-called intermediate configuration are preserved. The model is geometrically nonlinear and allows for finite elastic rotations, finite plastic deformations and overall finite deformations but remains a truly 'physically linear' theory as far as the elastic behaviour is concerned in the sense that simple uniaxial tension is modelled as linear and without viscosity.

We need to mention, however, that the new model is intrinsically rate-dependent, i.e., it is not possible to 'freeze' the 'visco-elastic' rotations $\bar{R}_{e}$ and obtain a frameindifferent reduced plasticity model. In other words, the used elastic-free energy $W$ is not expressible as a reduced function of $C=F^{T} F$. Nevertheless, the model is observerinvariant and the common wisdom that observer invariance implies a representation in $C$ or $U$ applies as such only to intrinsically nondissipative problems [42, p. 203]. In general, form invariance under superposed time-dependent rigid rotations (frame-indifference) implies observer invariance but is not identical to it. For this subtle point compare also to the lucid discussion in [29, p. 269] and [34, p. 159] together with [72, 4, 49, 50, 2

Let us now introduce the considered 3D-model which we have modified compared to [57, 53] to include also in a consistent manner "compressible" plasticity, i.e., $\operatorname{det}\left[F_{p}\right] \neq 1$. In the quasistatic setting appropriate for slow loading, where we neglect consistently inertia terms, we are led to study the following coupled minimization and evolution problem for the finite deformation $\varphi:[0, T] \times \bar{\Omega} \mapsto \mathbb{R}^{3}$, the plastic deformation $F_{p}$ : $[0, T] \times \bar{\Omega} \mapsto \mathrm{GL}^{+}(3, \mathbb{R})$ and the independent local visco-elastic rotation $\bar{R}_{e}:[0, T] \times \bar{\Omega} \mapsto$

\footnotetext{
${ }^{2}$ Also the undisputed physical principle is observer invariance and not directly frameindifference or form-invariance. The strengthening of form-invariance of the equations under superposed rigid rotations to form-invariance under the group of all diffeomorphisms is called covariance [42]. It is to be understood that form-invariance and covariance are additional constitutive assumptions. Interestingly, giving up the unnecessary frame-indifference allows us to show local existence.
} 
$\mathrm{SO}(3)$ on $\Omega$ :

$$
\begin{aligned}
I\left(\varphi, F_{p}^{-1}, \bar{R}_{e}\right) & =\int_{\Omega} W\left(F_{e}, \bar{R}_{e}\right) \operatorname{det}\left[F_{p}\right]-\langle f, \varphi\rangle \operatorname{det}\left[F_{p}\right] \mathrm{dV} \\
& -\int_{\Gamma_{S}}\langle N, \varphi\rangle\left\|\operatorname{Cof} F_{p} . \vec{n}\right\| \mathrm{dS} \mapsto \min . \text { w.r.t. } \varphi \text { at constant }\left(\bar{R}_{e}, F_{p}\right),
\end{aligned}
$$

with the Dirichlet boundary condition of place for the deformation $\varphi$ on $\Gamma \subset \partial \Omega: \varphi_{\left.\right|_{\Gamma}}=$ $g(t)$ and natural boundary conditions on $\partial \Omega \backslash \Gamma$. The corresponding field equations are

$$
0=\operatorname{Div}\left[D_{F}\left[W\left(F_{e}, \bar{R}_{e}\right) \operatorname{det}\left[F_{p}\right]\right]\right]+f \operatorname{det}\left[F_{p}\right] .
$$

The constitutive assumptions on the density are

$$
\begin{aligned}
W\left(F_{e}, \bar{R}_{e}\right) & =\frac{\mu}{4}\left\|F_{e}^{T} \bar{R}_{e}+\bar{R}_{e}^{T} F_{e}-2 \mathbb{1}\right\|^{2}+\frac{\lambda}{8} \operatorname{tr}\left[F_{e}^{T} \bar{R}_{e}+\bar{R}_{e}^{T} F_{e}-2 \mathbb{1}\right]^{2} \\
& =\mu\left\|\operatorname{sym}\left(\bar{U}_{e}-\mathbb{1}\right)\right\|^{2}+\frac{\lambda}{2} \operatorname{tr}\left[\operatorname{sym}\left(\bar{U}_{e}-\mathbb{1}\right)\right]^{2}, \bar{U}_{e}:=\bar{R}_{e}^{T} F_{e}, \quad F_{e}=\nabla \varphi \cdot F_{p}^{-1} .
\end{aligned}
$$

The coupled time evolution is given by

$$
\begin{aligned}
\frac{\mathrm{d}}{\mathrm{dt}}\left[F_{p}^{-1}\right] & \in-F_{p}^{-1} \cdot \partial \chi\left(\Sigma_{E}\right), \Sigma_{E}=F_{e}^{T} D_{F_{e}} W\left(F_{e}, \bar{R}_{e}\right) \operatorname{det}\left[F_{p}\right]-W\left(F_{e}, \bar{R}_{e}\right) \operatorname{det}\left[F_{p}\right] \mathbb{1}, \\
\frac{\mathrm{d} \hat{\omega}}{\mathrm{dt}} \bar{R}_{e}(t) & =\nu^{+} \operatorname{skew}(B) \cdot \bar{R}_{e}(t), \quad B=B_{\mathrm{mech}} \quad \text { or } \quad B_{\mathrm{tc}}, \quad \nu^{+}=\nu^{+}\left(F_{e}, \bar{R}_{e}\right) \in \mathbb{R}^{+}, \\
B_{\text {mech }} & =\mu F_{e} \bar{R}_{e}^{T}, \quad B_{\mathrm{tc}}=\left[\mu\left(2 \mathbb{1}-F_{e} \bar{R}_{e}^{T}\right)+\lambda\left[3-\left\langle F_{e} \bar{R}_{e}^{T}, \mathbb{1}\right\rangle\right]\right] F_{e} \bar{R}_{e}^{T},
\end{aligned}
$$

with initial conditions

$$
F_{p}^{-1}(0)=F_{p_{0}}^{-1}, \quad F_{p_{0}} \in \mathrm{GL}^{+}(3, \mathbb{R}), \quad \bar{R}_{e}(0)=\bar{R}_{e}^{0}, \quad \bar{R}_{e}^{0} \in \mathrm{SO}(3), \bar{R}_{e}^{0}=\mathbb{1} \text { if } F_{p_{0}}=\nabla \Theta .
$$

Here we specified $\mathfrak{f}=\partial \chi$, with the plastic flow potential $\chi: \mathbb{M}^{3 \times 3} \mapsto \mathbb{R}$, governing the plastic evolution and motivated through the principle of maximal dissipation sufficient for the thermodynamical consistency of the model $3^{3}$ The dead load body force and the boundary tractions are denoted by $f, N$, respectively, and defined w.r.t. the intermediate plastic configuration $F_{p}$ and $\vec{n}$ is the unit outward normal to $\partial \Omega$. Here $\Sigma_{E}$ denotes the elastic Eshelby stress tensor (the driving force behind evolving inhomogeneities in the reference configuration [45, 44]) which may be reduced to $\Sigma_{M}=F_{e}^{T} D_{F_{e}} W\left(F_{e}, \bar{R}_{e}\right)$, the elastic Mandel stress tensor in case of a deviatoric flow rule which preserves the incompressibility constraint $\operatorname{det}\left[F_{p}\right]=1$. This is a generalization of the gradient type models in the sense of [1] to finite deformations.

By $\frac{d_{\omega}}{d t}$ we mean the observer invariant time derivative on $\operatorname{SO}(3, \mathbb{R})$

$$
\frac{\mathrm{d}_{\hat{\omega}}}{\mathrm{dt}}[R(t)]:=\frac{\mathrm{d}}{\mathrm{dt}}[R(t)]-\hat{\omega}(t) \cdot R(t), \quad \hat{\omega}:=\frac{\mathrm{d}}{\mathrm{dt}}[Q(t)] \cdot Q(t)^{T},
$$

where $Q(t) \in \mathrm{SO}(3, \mathbb{R})$ is the instantaneous rotation of the current frame with respect to the inertial frame and $\hat{\omega}$ is the corresponding angular velocity. Without loss of generality we confine our attention to the inertial frame, i.e., $\hat{\omega} \equiv 0$ and $\frac{\mathrm{d}_{\hat{\omega}}}{\mathrm{dt}}=\frac{\mathrm{d}}{\mathrm{dt}}$.

\footnotetext{
${ }^{3}$ This leads to an associative formulation. However, for our mathematical development, this specification is not strictly necessary. We can deal as well with nonassociative formulations.
} 
The term $\nu^{+}:=\frac{1}{\eta_{e}} \nu^{+}\left(F_{e}, \bar{R}_{e}\right)$ represents a scalar-valued function introducing elastic viscosity within the elastic domain and $\eta_{e}$ plays the role of a relaxation time with units $\left[\eta_{e}\right]=$ sec. Here $F_{p_{0}}^{-1}$ and $\bar{R}_{e}^{0}$ are the initial conditions for the plastic deformation and visco-elastic rotation part, respectively. The choice $B=B_{\mathrm{tc}}$ is fully thermodynamically consistent, whereas the simpler choice $B=B_{\text {mech }}$ is (only) mechanically consistent in the sense that various invariance requirements are met. 4

Due to the underlying isotropy the resulting model (P3) with $B=B_{\text {mech }}$ approaches in the equilibrium limit $\nu^{+} \rightarrow \infty$ (vanishing elastic viscosity $=$ zero relaxation limit $\eta_{e} \rightarrow 0$ viz. for arbitrary slow processes) formally the problem

$$
\begin{aligned}
\int_{\Omega} W_{\infty}\left(F_{e}\right) \operatorname{det}\left[F_{p}\right]-\langle f, \varphi\rangle \operatorname{det}\left[F_{p}\right] \mathrm{dV} & \\
& -\int_{\Gamma_{S}}\langle N, \varphi\rangle\left\|\operatorname{Cof} F_{p} \cdot \vec{n}\right\| \mathrm{dS} \mapsto \text { stat. w.r.t. } \varphi \text { at constant } F_{p}, \\
W_{\infty}\left(F_{e}\right) & =\mu\left\|U_{e}-\mathbb{1}\right\|^{2}+\frac{\lambda}{2} \operatorname{tr}\left[U_{e}-\mathbb{1}\right]^{2}, \quad F_{e}=\nabla \varphi F_{p}^{-1}, U_{e}=R_{e}^{T} F_{e}, \\
\frac{\mathrm{d}}{\mathrm{dt}}\left[F_{p}^{-1}\right](t) & \in-F_{p}^{-1}(t) \cdot \partial \chi\left(\Sigma_{E, \infty}\right), \\
\Sigma_{E, \infty} & =U_{e} D_{U_{e}} W_{\infty}\left(U_{e}\right) \operatorname{det}\left[F_{p}\right]-W_{\infty}\left(U_{e}\right) \operatorname{det}\left[F_{p}\right] \mathbb{1},
\end{aligned}
$$

with $U_{e}=\left(F_{e}^{T} F_{e}\right)^{\frac{1}{2}}$ the symmetric elastic stretch, $U_{e}-\mathbb{1}$ the elastic Biot strain tensor and $W_{\infty}$ the nonelliptic equilibrium energy. The system (2.9) is an exact equilibrium model for small elastic strains and finite plastic deformations in the classical sense with no internal dissipation due to visco-elastic effects. The transition from (P3) to (2.9) is not entirely trivial since it is not just the replacement of $\bar{R}_{e}$ by $R_{e}=\operatorname{polar}\left(F_{e}\right)$ and note the subtle change from global minimization to a stationarity requirement only. Observe as well that $\mu\|U-\mathbb{1}\|^{2}+\frac{\lambda}{2} \operatorname{tr}[U-\mathbb{1}]^{2}$ leads to a linear response in uniaxial tension while, e.g., $\mu\|E\|^{2}+\frac{\lambda}{2} \operatorname{tr}[E]^{2}$ leads to a nonlinear, nonphysical response in uniaxial tension.

In the companion paper [57] the implications, predictions and physical relevance of the new model have been investigated in great detail. It is shown that the additional degrees of freedom inherent through the independent local "visco-elastic" rotations $\bar{R}_{e}$ can be interpreted in the framework of a material with a polycrystalline structure where the averaged individual rotations of the grains may deviate from the continuum rotation. 5 Then, in the presence of plasticity, $\bar{R}_{e}$ represents a reversible, 'visco-elastic' part of the total rotation of the grains and leads to texture effects (deformation induced

\footnotetext{
${ }^{4}$ Under the simplifying assumptions $F=\mathbb{1}+\nabla u, F_{p}=\mathbb{1}+p, \bar{R}_{e}=\mathbb{1}+\bar{A}_{e}, \bar{A}_{e} \in \mathfrak{s o}(3, \mathbb{R})$ with $\nabla u, p, \bar{A}_{e} \ll 1$ the finite model $(\mathrm{P} 3$ reduces formally in first order to the infinitesimal elastic-plastic model [2.4] with $\varepsilon_{p}:=\operatorname{sym} p$. Hence, grain boundary relaxation in our terminology is a second order effect only. In contrast, visco-elasticity is traditionally introduced as a first order effect, already effective in infinitesimal elasticity. Furthermore, our additional elastic viscosity is only operative in torsion, not in tension!

${ }^{5}$ Observe that these two rotations do not coincide in general: the averaged rotation is understood to be the best-approximating single rotation to a rotation field defined over a representative volume element while the continuum rotation is the orthogonal part of the averaged deformation gradient. In the infinitesimal case, both infinitesimal rotations would coincide!
} 
anisotropy). The evolution equation for $\bar{R}_{e}$ introduces hysteresis effects into the model already within the elastic region, i.e., immediately for arbitrary small stress levels. The physical reality of this behaviour for polycrystalline material is well documented and it is shown that the new model (P3) allows a qualitative and in part quantitative description of such effects which are ascribed to internal friction at the grain boundaries. In [57] it has also been intimated that the elastic viscosity is larger for larger internal surfaces, i.e., the smaller the grain size, while single crystals behave nearly rate-independent for that matter. Let us define $\hat{g}$ to be the average grain diameter and $\hat{L}$ to be the edge length of a representative volume element. Then the internal surface in such a volume element scales like $\left|S_{\text {int }}^{3 d}\right| \sim \frac{\hat{L}^{3}}{\hat{g}^{3}}$. This translates into the additional constitutive requirement

$$
\nu_{e}^{+} \sim \frac{1}{\left|S_{\text {int }}^{3 d}\right|} \sim \frac{\hat{g}^{3}}{\hat{L}^{3}}
$$

where $\left|S_{\text {int }}^{3 d}\right|$ is a characteristic measure of the internal surface for a polycrystalline structure. Therefore, the elastic viscosity $\nu_{e}^{+}$is related to an internal characteristic length.

In the mathematical part of this contribution we will show the following result:

TheOREm 2.1 (Local existence and uniqueness for problem (P3)). Let $\Omega \subset \mathbb{R}^{3}$ be a bounded smooth domain. Furthermore, suppose for the displacement boundary data $g \in$ $C^{1}\left(\mathbb{R}, H^{3,2}\left(\Omega, \mathbb{R}^{3}\right)\right)$ and for the body force $f \in C^{1}\left(\mathbb{R}, H^{1,2}\left(\Omega, \mathbb{R}^{3}\right)\right)$. Assume for the initial conditions $\left(F_{p}^{-1,0}, \bar{R}_{e}^{0}\right) \in H^{2,2}(\Omega, \mathrm{SL}(3, \mathbb{R})) \times H^{2,2}(\Omega, \mathrm{SO}(3))$. Then there exists a time $t_{1}>0$ such that the initial boundary value problem (P3) in its viscoelastic-viscoplastic form 3.31 and 3.32 admits a unique solution

$$
\left(\varphi, F_{p}, \bar{R}_{e}\right) \in C\left(\left[0, t_{1}\right], H^{3,2}\left(\Omega, \mathbb{R}^{3}\right)\right) \times C^{1}\left(\left[0, t_{1}\right], H^{2,2}(\Omega, \mathrm{SL}(3, \mathbb{R})), H^{2,2}(\Omega, \mathrm{SO}(3))\right) .
$$

REMARK 2.2 (Smoothness and infinitesimal models). The foregoing existence result sheds some light on the physical relevance of global results for weak solutions of infinitesimal elasto-plasticity models of the type (2.4). Typically one arrives at global weak solutions with $\varepsilon, \varepsilon_{p} \in L^{\infty}\left(\mathbb{R}^{+}, L^{2}(\Omega, \mathfrak{s l}(3, \mathbb{R}))\right)$, at best. To obtain these results, decisive use is made of inequality 2.17) with $\mathbb{D}_{\text {lin }}$ constant. However, $\varepsilon_{p} \in L^{\infty}\left(\mathbb{R}^{+}, L^{2}(\Omega, \mathfrak{s l}(3, \mathbb{R}))\right)$ conceptually corresponds to $F_{p} \in L^{\infty}\left(\mathbb{R}^{+}, L^{2}(\Omega, \operatorname{SL}(3, \mathbb{R}))\right)$ under which condition alone coercivity of the geometrically exact elastic problem is not true. The infinitesimal model practically rules out elastic failure of the material due to fatigue. Since, however, fatigue is an important ingredient of plasticity theory, the possibility of fatigue has to be reintroduced artificially in infinitesimal continuum damage models [31. We see that the model (I3) provides a much more realistic view of the plasticity of polycrystals.

2.4. General mathematical framework. Let us outline how we show that (P3) admits a unique local solution. At 'frozen' variables $\left(F_{p}, \bar{R}_{e}\right)$ the above system of elastic balance of linear momentum (P3.1) proves to be a linear, second order, strictly LegendreHadamard elliptic boundary value problem with nonconstant coefficients. This system has variational structure in the sense that the equilibrium part (P3.1) of (P3) is formally equivalent to the elastic minimization problem (without loss of generality we assume for 
the mathematics $\left.F_{p} \in \mathrm{SL}(3, \mathbb{R})\right)$

$$
\forall t \in[0, T]: \quad I\left(\varphi(t), F_{p}^{-1}(t), \bar{R}_{e}(t)\right) \mapsto \min . \text { w.r.t. } \quad \varphi(t) \in g(t)+H_{\circ}^{1,2}(\Omega, \Gamma),
$$

where

$$
I\left(\varphi, F_{p}^{-1}, \bar{R}_{e}\right):=\int_{\Omega} W\left(\nabla \varphi F_{p}^{-1}, \bar{R}_{e}\right)-\langle f, \varphi\rangle \mathrm{dV} .
$$

The weak form of the corresponding equilibrium equation is given next.

LEMma 2.3 (Weak form of elastic problem). If a minimizer $\varphi \in H^{1,2}(\Omega)$ of (2.11) exists, then it is a weak solution to the equilibrium problem

$$
0=\int_{\Omega}\left\langle D_{F}\left[W\left(\nabla \varphi F_{p}^{-1}, \bar{R}_{e}\right)\right], \nabla \phi\right\rangle-\langle f, \phi\rangle \mathrm{dV} \quad \forall \phi \in H_{0}^{1,2}\left(\Omega, \mathbb{R}^{3}\right),
$$

which satisfies the corresponding strong form

$$
\begin{aligned}
\operatorname{Div} & \bar{R}_{e}\left[\mu\left(\left(\nabla \varphi F_{p}^{-1}\right)^{T} \bar{R}_{e}+\bar{R}_{e}^{T} \nabla \varphi F_{p}^{-1}\right)+\lambda \operatorname{tr}\left[\left(\nabla \varphi F_{p}^{-1}\right)^{T} \bar{R}_{e}\right] \mathbb{1}\right] F_{p}^{-T} \\
& =-f+\operatorname{Div}\left[(2 \mu+3 \lambda) \bar{R}_{e} F_{p}^{-T}\right],
\end{aligned}
$$

if the appearing quantities are smooth enough.

Observe in passing that for $F_{p}=\mathbb{1}, \bar{R}_{e}=\mathbb{1}$ and with $\operatorname{Div}\left(\nabla u^{T}\right)=\nabla(\operatorname{Div} u)$ and $\operatorname{Div}((\operatorname{Div} u) \cdot \mathbb{1})=\nabla(\operatorname{Div} u)$ we recover naturally the Lamé equations

$$
(\mu+\lambda) \nabla(\operatorname{Div} u)+\mu \Delta u+f=0
$$

of linearized elasticity. We note also the appearance of a "virtual" body force contribution due to the inhomogeneities inherent in $F_{p}, \bar{R}_{e}$. This equilibrium system (2.13) can be written in the short form

$$
\operatorname{Div} \mathbb{D}(A(x)) . \nabla \varphi=f+\operatorname{Div} V(A), \quad \varphi_{\left.\right|_{\Gamma}}=g,
$$

where we have set $A=\left(F_{p}^{-T}, \bar{R}_{e}\right)$ and introduced the corresponding elasticity tensor $\mathbb{D}$ and the additional right-hand side contribution $V$ according to the next definition in line with equation (2.13).

Definition 2.4 (Corresponding elasticity tensor). We define the elasticity tensor $\mathbb{D}: \mathbb{M}^{3 \times 3} \times \mathbb{M}^{3 \times 3} \mapsto \operatorname{Lin}\left(\mathbb{M}^{3 \times 3}, \mathbb{M}^{3 \times 3}\right)$ and the right-hand side $V: \mathbb{M}^{3 \times 3} \times \mathbb{M}^{3 \times 3} \mapsto \mathbb{M}^{3 \times 3}$ by setting $\forall H \in \mathbb{M}^{3 \times 3}$ :

$$
\begin{aligned}
& \mathbb{D}\left(F_{p}^{-T}, \bar{R}_{e}\right) \cdot H:=\bar{R}_{e}\left[\mu\left(\left(H F_{p}^{-1}\right)^{T} \bar{R}_{e}+\bar{R}_{e}^{T} H F_{p}^{-1}\right)+\lambda \operatorname{tr}\left[\left(H F_{p}^{-1}\right)^{T} \bar{R}_{e}\right] \mathbb{1}\right] F_{p}^{-T}, \\
& V\left(F_{p}^{-T}, \bar{R}_{e}\right):=(2 \mu+3 \lambda) \bar{R}_{e} F_{p}^{-T},
\end{aligned}
$$

respectively.

We are then concerned with the static situation where $A=\left(F_{p}^{-T}, \bar{R}_{e}\right)$ is assumed to be known.

A startling difficulty we encounter is that the elasticity tensor $\mathbb{D}=\mathbb{D}(A)$, although turning out to be uniformly Legendre-Hadamard elliptic, does not induce a pointwise uniformly positive bilinear form. Such a problem does not appear in infinitesimal elastoviscoplasticity since there the relevant elasticity tensor $\mathbb{D}_{\operatorname{lin}} \in \operatorname{Lin}(\operatorname{Sym}(3)$, $\operatorname{Sym}(3))$ is 
assumed to be a constant uniformly positive definite fourth order tensor defined on the symmetrized strains $\varepsilon$ such that

$$
\forall \varepsilon \in \operatorname{Sym}(3): \quad\left\langle\mathbb{D}_{\operatorname{lin}} \cdot \varepsilon, \varepsilon\right\rangle \geq c^{+} \cdot\|\varepsilon\|^{2},
$$

and coercivity follows from the standard Korn first inequality [16].

Nevertheless, we prove the existence, uniqueness and regularity of solutions to the boundary value problem (P3.1). The existence part now relies heavily on Theorem 6.2 recently proved by the author extending Korn's first inequality to nonconstant coefficients and overcoming the lack of uniform positivity of (2.11). This theorem has been proved precisely with the motivation of applying it to finite plasticity.

Now, again in stark contrast to the infinitesimal case $(2.4)_{1}$ with additive decomposition, where the solution $u$ depends linearly on the plastic strain $\varepsilon_{p}$, the solution of (2.13) depends nonlinearly on $A$ due to the underlying multiplicative structure.

Despite this nonlinearity, we establish Lipschitz-continuous dependence of the solution to (2.11) with respect to the data and coefficients $A=\left(F_{p}^{-T}, \bar{R}_{e}\right)$ by looking at the system (2.13) in the form (2.15) and using sharp elliptic estimates. Since the constant in the extended Korn inequality Theorem 6.2 enters the Lipschitz estimate but is itself determined in a nonconstructive way, we show that this constant can be bounded below for bounded, smooth coefficients.

The conceptual idea of treating the coupled plastic evolution problem is then straightforward: in the fully rate-dependent case the differential inclusion is in fact an ordinary differential equation which may be written in the form

$$
\frac{\mathrm{d}}{\mathrm{dt}} A(t)=\mathfrak{h}(\nabla \varphi(A), A) \cdot A,
$$

with $\mathfrak{h}: \mathbb{M}^{3 \times 3} \times\left(\mathbb{M}^{3 \times 3} \times \mathbb{M}^{3 \times 3}\right) \mapsto \operatorname{Lin}\left(\mathbb{M}^{3 \times 3}, \mathbb{M}^{3 \times 3}\right)$, where $A=\left(F_{p}^{-T}, \bar{R}_{e}\right)$ and $\varphi=\varphi(A)$ is the solution of the elliptic boundary value problem (P3.1) at given $A$. It remains to show that the right-hand side of (2.18) as a function of $A$ is locally Lipschitz continuous allowing us to apply the local existence and uniqueness theorem for nonlinear evolution equations in Banach spaces based on Banach's fixed point theorem; cf. Theorem 6.1.

The presented formal mathematical framework has a natural counterpart in the numerical treatment of visco-plasticity. The coupled problem can be considered to be a differential algebraic system of equations (DAE) of index 1; the algebraic equation is the side condition coming from a discretization of the elastic equilibrium system [23]. That viscosity is indeed enough to regularize problems in finite plasticity is a standing conjecture [52] and is rigorously justified by our development.

\section{Local existence and uniqueness proof.}

3.1. Definitions and assumptions. To streamline the subsequent mathematical investigation of (2.13) and to place it in a more general context, we introduce the following definitions.

Definition 3.1 (General assumption, GA). (GA.1) $\Omega \subset \mathbb{R}^{n}$ is a bounded domain with smooth boundary and space dimension $n$.

(GA.2) We call $m \in \mathbb{N}$ the order of elliptic regularity and assume $2 \cdot(m+1)>n$. 
(GA.3)(Local boundedness of the elasticity tensor and part of the right-hand side) For given $K_{1}>0$ :

$$
\begin{aligned}
& \mathbb{D}: \mathbb{M}^{3 \times 3} \times \mathbb{M}^{3 \times 3} \mapsto \operatorname{Lin}\left(\mathbb{M}^{3 \times 3}, \mathbb{M}^{3 \times 3}\right), \quad V: \mathbb{M}^{3 \times 3} \times \mathbb{M}^{3 \times 3} \mapsto \mathbb{M}^{3 \times 3}, \\
& \mathcal{M}:=\left\{A: \Omega \mapsto \mathbb{M}^{3 \times 3} \times \mathbb{M}^{3 \times 3} \mid\|A\|_{m+1,2, \Omega} \leq K_{1}\right\}, \\
& \exists C_{\mathcal{M}}: \quad \forall A \in \mathcal{M}: \quad\|\mathbb{D}(A)\|_{m+1,2, \Omega},\|V(A)\|_{m+1,2, \Omega} \leq C_{\mathcal{M}} .
\end{aligned}
$$

(GA.4) (Uniform Legendre-Hadamard ellipticity on $\mathcal{M}$ ) For all $\xi, \eta \in \mathbb{R}^{3}$ it holds that

$$
\exists c_{e, \mathcal{M}}^{+}>0: \forall x \in \Omega: \forall A \in \mathcal{M}: \quad\langle\mathbb{D}(A(x)) .(\xi \otimes \eta), \xi \otimes \eta\rangle \geq c_{e, \mathcal{M}}^{+} \cdot\|\xi\|^{2}\|\eta\|^{2} .
$$

(GA.5) (Local Lipschitz continuity)

$$
\begin{array}{ll}
\exists L_{\mathcal{M}}: \forall A, B \in \mathcal{M}: & \|\mathbb{D}(A)-\mathbb{D}(B)\|_{m+1,2, \Omega} \leq L_{\mathcal{M}} \cdot\|A-B\|_{m+1,2, \Omega}, \\
\exists L_{\mathcal{M}}: \forall A, B \in \mathcal{M}: & \|V(A)-V(B)\|_{m+1,2, \Omega} \leq L_{\mathcal{M}} \cdot\|A-B\|_{m+1,2, \Omega} .
\end{array}
$$

If (GA.1)-(GA.5) hold, we say that GA holds. Note that condition (GA.5) already implies (GA.3) but for convenience (GA.3) is stated separately.

3.2. Existence of weak solutions to the elastic subproblem. We already indicated that in the static case for frozen variables $\left(F_{p}, \bar{R}_{e}\right)$ the elastic equilibrium system ( (3) is a linear, strictly Legendre-Hadamard elliptic second order boundary value problem with nonconstant coefficients and variational structure 6 We exploit this structure and apply the direct methods of the calculus of variations to show that there exists a unique weak solution at frozen variables $\left(F_{p}, \bar{R}_{e}\right)$.

ThEOREM 3.2 (Existence of minimizers). Let $F_{p}, F_{p}^{-1} \in H^{2,2}(\Omega, \mathrm{GL}(3, \mathbb{R}))$ and $\bar{R}_{e} \in$ $H^{2,2}(\Omega, \mathrm{SO}(3))$ be given. Assume for the Dirichlet boundary data $g \in H^{3,2}(\Omega)$ and for the body force $f \in L^{2}(\Omega)$. Then the variational problem

$$
\begin{aligned}
& I\left(\varphi, F_{p}^{-1}, \bar{R}_{e}\right) \mapsto \min ., \quad \varphi \in g+H_{\circ}^{1,2}(\Omega), \\
& I\left(\varphi, F_{p}^{-1}, \bar{R}_{e}\right)=\int_{\Omega} W\left(\nabla \varphi F_{p}^{-1}, \bar{R}_{e}\right)-\langle f, \varphi\rangle_{\mathbb{R}^{3}} \mathrm{dV}
\end{aligned}
$$

admits a minimizer $\varphi \in H^{1,2}(\Omega)$ and this minimizer satisfies estimate (3.13).

Proof. By the definition of $I$ as a sum of a quadratic form and a linear form it is easy to see that $\varphi \mapsto I\left(\varphi, F_{p}^{-1}, \bar{R}_{e}\right)$ is convex over $H^{1,2}(\Omega)$. Moreover with $g \in H^{1,2}(\Omega)$ we have that $I\left(g, F_{p}^{-1}, \bar{R}_{e}\right)<\infty$. First we show that $I$ is bounded below. To this end define $\hat{F}_{p}=\bar{R}_{e} \cdot F_{p}$. Note that $\hat{F}_{p} \in H^{2,2}(\Omega)$ since $H^{2,2}(\Omega)$ is an algebra for $n=3$ space dimensions. Then

$$
\begin{aligned}
W\left(F_{e}, \bar{R}_{e}\right) & =\frac{\mu}{4}\left\|F_{e}^{T} \bar{R}_{e}+\bar{R}_{e}^{T} F_{e}-2 \mathbb{1}\right\|^{2}+\frac{\lambda}{8} \operatorname{tr}\left[F_{e}^{T} \bar{R}_{e}+\bar{R}_{e}^{T} F_{e}-2 \mathbb{1}\right]^{2} \\
& =\frac{\mu}{4}\left\|\hat{F}_{p}^{-T} \nabla \varphi^{T}+\nabla \varphi \hat{F}_{p}^{-1}-2 \mathbb{1}\right\|^{2}+\frac{\lambda}{8} \operatorname{tr}\left[\hat{F}_{p}^{-T} \nabla \varphi^{T}+\nabla \varphi \hat{F}_{p}^{-1}-2 \mathbb{1}^{2}\right.
\end{aligned}
$$

\footnotetext{
${ }^{6}$ This is essentially the elastic trial step in current algorithmic formulations.
} 
where we used that $\left\|\bar{R}_{e} X \bar{R}_{e}^{T}\right\|=\|X\|$ and $\operatorname{tr}\left[\bar{R}_{e} X \bar{R}_{e}^{T}\right]=\operatorname{tr}[X]$. Now set $\varphi=v+g$ with $v \in H_{\circ}^{1,2}(\Omega)$. We have algebraically

$$
\begin{aligned}
& W\left(\nabla \varphi F_{p}^{-1}, \bar{R}_{e}\right) \\
& =\frac{\mu}{4}\left\|\hat{F}_{p}^{-T} \nabla \varphi^{T}+\nabla \varphi \hat{F}_{p}^{-1}-2 \mathbb{1}\right\|^{2}+\frac{\lambda}{8} \operatorname{tr}\left[\hat{F}_{p}^{-T} \nabla \varphi^{T}+\nabla \varphi \hat{F}_{p}^{-1}-2 \mathbb{1}\right]^{2} \\
& \geq \frac{\mu}{4}\left\|\hat{F}_{p}^{-T} \nabla v^{T}+\nabla v \hat{F}_{p}^{-1}\right\|^{2}-2 \mu\left\|\hat{F}_{p}^{-1}\right\|^{2}\|\nabla v\|\|\nabla g\|-2 \mu \sqrt{3}\left\|\hat{F}_{p}^{-1}\right\|\|\nabla v\| \\
& \quad+\frac{\mu}{4}\left\|\hat{F}_{p}^{-T} \nabla g^{T}+\nabla g \hat{F}_{p}^{-1}-2 \mathbb{1}\right\|^{2} .
\end{aligned}
$$

Integrating over $\Omega$ and making use of Theorem 6.2 in the improved version of 63 with $\hat{F}_{p}, \hat{F}_{p}^{-1} \in H^{2,2}(\Omega) \subset C^{0, \frac{1}{2}}(\bar{\Omega})$, we get for all $\varphi \in H^{1,2}(\Omega)$

$$
\begin{aligned}
& \int_{\Omega} W\left(\nabla \varphi F_{p}^{-1}, \bar{R}_{e}\right)-\langle f, \varphi\rangle \mathrm{dV} \geq \mu \underbrace{c^{+}\left(\hat{F}_{p}\right)\|v\|_{1,2, \Omega}^{2}}_{\text {extended Korn }}-2 \mu\left\|\hat{F}_{p}^{-1}\right\|_{\infty}^{2}\|\nabla g\|_{\infty}\|v\|_{1,2, \Omega} \\
& -2 \mu \sqrt{3}\left\|\hat{F}_{p}^{-1}\right\|_{\infty}\|v\|_{1,2, \Omega}+\int_{\Omega} \frac{\mu}{4}\left\|\hat{F}_{p}^{-T} \nabla g^{T}+\nabla g \hat{F}_{p}^{-1}-2 \mathbb{1}\right\|^{2} \mathrm{dV} \\
& \quad-\|f\|_{2, \Omega}\left(\|v\|_{2, \Omega}+\|g\|_{2, \Omega}\right) .
\end{aligned}
$$

Hence $I$ is bounded below and we observe that a minimizing sequence $\left\{\varphi_{n}\right\}_{n=1}^{\infty} \subset H^{1,2}(\Omega)$ exists with

$$
I\left(\varphi_{n}, F_{p}^{-1}, \bar{R}_{e}\right) \rightarrow \inf _{\varphi \in g+H_{\circ}^{1,2}(\Omega)} I\left(\varphi, F_{p}^{-1}, \bar{R}_{e}\right), \quad n \rightarrow \infty
$$

We proceed to show that $I$ is coercive which implies that $\left\{\varphi_{n}\right\}_{n=1}^{\infty}$ is bounded.

Our development is more detailed than strictly necessary if only coercivity were concerned. However, we need to keep track of all constants for uniform estimates in subsequent paragraphs.

Without loss of generality assume that $\varphi_{1}=g$. Since $\varphi_{n}$ is a minimizing sequence, we have by estimating from above and using $\langle X, \mathbb{1}\rangle^{2} \leq 3\|X\|^{2}$

$$
\begin{aligned}
& \int_{\Omega} W\left(\nabla \varphi_{n} F_{p}^{-1}, \bar{R}_{e}\right)-\left\langle f, \varphi_{n}\right\rangle \mathrm{dV} \\
& \leq\left(\frac{\mu}{4}+\frac{3 \lambda}{8}\right) \int_{\Omega}\left\|\hat{F}_{p}^{-T} \nabla g^{T}+\nabla g \hat{F}_{p}^{-1}-2 \mathbb{1}\right\|^{2} \mathrm{dV}+\|f\|_{2, \Omega}\|g\|_{2, \Omega} \\
& \leq \frac{\mu}{4} \int_{\Omega}\left\|\hat{F}_{p}^{-T} \nabla g^{T}+\nabla g \hat{F}_{p}^{-1}-2 \mathbb{1}\right\|^{2} \mathrm{dV} \\
& \quad+\frac{3 \lambda}{2}|\Omega|\left(\left\|\hat{F}_{p}^{-1}\right\|_{\infty}^{2}\|\nabla g\|_{\infty}^{2}+2 \sqrt{3}\left\|\hat{F}_{p}^{-1}\right\|_{\infty}\|\nabla g\|_{\infty}+3\right)+\|f\|_{2, \Omega}\|g\|_{2, \Omega}
\end{aligned}
$$


This implies together with (3.4) the inequality

$$
\begin{aligned}
& \frac{3 \lambda}{2}|\Omega|\left(\left\|\hat{F}_{p}^{-1}\right\|_{\infty}^{2}\|\nabla g\|_{\infty}^{2}+2 \sqrt{3}\left\|\hat{F}_{p}^{-1}\right\|_{\infty}\|\nabla g\|_{\infty}+3\right)+2\|f\|_{2, \Omega}\|g\|_{2, \Omega} \\
& \geq \mu c^{+}\left(\hat{F}_{p}\right)\left\|v_{n}\right\|_{1,2, \Omega}^{2}-2 \mu\left\|\hat{F}_{p}^{-1}\right\|_{\infty}^{2}\|\nabla g\|_{\infty}\left\|v_{n}\right\|_{1,2, \Omega} \\
& \quad-2 \mu \sqrt{3}\left\|\hat{F}_{p}^{-1}\right\|_{\infty}\left\|v_{n}\right\|_{1,2, \Omega}-\|f\|_{2, \Omega}\left\|v_{n}\right\|_{2, \Omega} \\
& \geq \mu c^{+}\left(\hat{F}_{p}\right)\left\|v_{n}\right\|_{1,2, \Omega}^{2}-2 \mu \sqrt{3}\left(1+\left\|\hat{F}_{p}^{-1}\right\|_{\infty}^{2}\right)\left[\left\|\hat{F}_{p}^{-1}\right\|_{\infty}+\|\nabla g\|_{\infty}+\|f\|_{2, \Omega}\right] \cdot\left\|v_{n}\right\|_{1,2, \Omega} .
\end{aligned}
$$

Hence a rough estimate yields

$$
\begin{aligned}
& 5 \lambda|\Omega|\left(1+\left\|\hat{F}_{p}^{-1}\right\|_{\infty}\|\nabla g\|_{\infty}\right)^{2}+2\|f\|_{2, \Omega}\|g\|_{2, \Omega} \\
& \geq \mu c^{+}\left(\hat{F}_{p}\right)\left\|v_{n}\right\|_{1,2, \Omega}^{2}-5 \mu\left(1+\left\|\hat{F}_{p}^{-1}\right\|_{\infty}^{2}\right)\left[\left\|\hat{F}_{p}^{-1}\right\|_{\infty}+\|\nabla g\|_{\infty}+\|f\|_{2, \Omega}\right] \cdot\left\|v_{n}\right\|_{1,2, \Omega}
\end{aligned}
$$

Thus we get a quadratic inequality in $\left\|v_{n}\right\|_{1,2, \Omega}$ :

$$
\begin{aligned}
0 \geq & \left\|v_{n}\right\|_{1,2, \Omega}^{2}-\frac{5}{c^{+}\left(\hat{F}_{p}\right)}\left(1+\left\|\hat{F}_{p}^{-1}\right\|_{\infty}^{2}\right)\left[\left\|\hat{F}_{p}^{-1}\right\|_{\infty}+\|\nabla g\|_{\infty}+\|f\|_{2, \Omega}\right] \cdot\left\|v_{n}\right\|_{1,2, \Omega} \\
& -\frac{5 \lambda|\Omega|}{\mu c^{+}\left(\hat{F}_{p}\right)}\left(1+\left\|\hat{F}_{p}^{-1}\right\|_{\infty}\|\nabla g\|_{\infty}\right)^{2}-2\|f\|_{2, \Omega}\|g\|_{2, \Omega}
\end{aligned}
$$

Since $0 \geq x^{2}-b x-c \Rightarrow x \leq b+\sqrt{c}$, the former yields

$$
\begin{aligned}
\left\|v_{n}\right\|_{1,2, \Omega} \leq & {\left[\frac{5}{c\left(\hat{F}_{p}\right)}\left(1+\left\|\hat{F}_{p}^{-1}\right\|_{\infty}^{2}\right)+\sqrt{\frac{5 \lambda|\Omega|}{\mu c\left(\hat{F}_{p}\right)}} \frac{\left(1+\left\|\hat{F}_{p}^{-1}\right\|_{\infty}\|\nabla g\|_{\infty}\right)}{\left\|\hat{F}_{p}^{-1}\right\|_{\infty}+\|\nabla g\|_{\infty}+\|f\|_{2, \Omega}}\right.} \\
+ & \left.\frac{\|f\|_{2, \Omega}+\|g\|_{2, \Omega}}{\left\|\hat{F}_{p}^{-1}\right\|_{\infty}+\|\nabla g\|_{\infty}+\|f\|_{2, \Omega}}\right] \cdot\left[\left\|\hat{F}_{p}^{-1}\right\|_{\infty}+\|\nabla g\|_{\infty}+\|f\|_{2, \Omega}\right] .
\end{aligned}
$$

Taking $\hat{F}_{p}^{-1}=F_{p}^{-1} \cdot \bar{R}_{e}^{T}$ into account and the estimate $\sqrt{3}=\|\mathbb{1}\|=\left\|F_{p}^{-1} F_{p}\right\| \leq$ $\left\|F_{p}^{-1}\right\|\left\|F_{p}\right\|$ (which implies $\left\|F_{p}^{-1}\right\| \geq \frac{\sqrt{3}}{\left\|F_{p}\right\|}$ ) shows

$$
\begin{aligned}
\left\|v_{n}\right\|_{1,2, \Omega} \leq & {\left[\frac{5}{c\left(\hat{F}_{p}\right)}\left(1+\left\|F_{p}^{-1}\right\|_{\infty}^{2}\right)+\sqrt{\frac{5 \lambda|\Omega|}{\mu c\left(\hat{F}_{p}\right)}}\left\|F_{p}\right\|_{\infty} \frac{\left(1+\left\|F_{p}^{-1}\right\|_{\infty}\|\nabla g\|_{\infty}\right)}{\sqrt{3}}\right.} \\
& \left.+\frac{\left\|F_{p}\right\|_{\infty}}{\sqrt{3}}\left(\|f\|_{2, \Omega}+\|g\|_{2, \Omega}\right)\right] \cdot\left[\left\|F_{p}^{-1}\right\|_{\infty}+\|\nabla g\|_{\infty}+\|f\|_{2, \Omega}\right] .
\end{aligned}
$$

With the embedding $H^{m, 2}(\Omega) \hookrightarrow C^{m-\frac{n}{2}}(\bar{\Omega})$ and adding $\left\|\bar{R}_{e}\right\|_{2,2, \Omega}$, we get the estimate $\left\|v_{n}\right\|_{1,2, \Omega}$

$$
\begin{aligned}
\leq & C(\Omega)\left[\frac{1}{c\left(\hat{F}_{p}\right)}\left(1+\left\|F_{p}^{-1}\right\|_{2,2, \Omega}^{2}\right)+\left\|F_{p}\right\|_{2,2, \Omega} \sqrt{\frac{\lambda|\Omega|}{\mu c\left(\hat{F}_{p}\right)}}\left(1+\left\|F_{p}^{-1}\right\|_{2,2, \Omega}\|g\|_{3,2, \Omega}\right)\right. \\
& \left.+\left\|F_{p}^{-1}\right\|_{2,2, \Omega}\left(\|f\|_{2, \Omega}+\|g\|_{2, \Omega}\right)\right] \cdot\left[\left\|F_{p}^{-1}\right\|_{2,2, \Omega}+\left\|\bar{R}_{e}\right\|_{2,2, \Omega}+\|g\|_{3,2, \Omega}+\|f\|_{2, \Omega}\right] .
\end{aligned}
$$


Therefore for $\varphi_{n}=v_{n}+g$ we get

$$
\begin{aligned}
& \left\|\varphi_{n}\right\|_{1,2, \Omega} \\
& \leq\left(1+C(\Omega)\left[\frac{1}{c\left(\hat{F}_{p}\right)}\left(1+\left\|F_{p}^{-1}\right\|_{2,2, \Omega}^{2}\right)+\left\|F_{p}\right\|_{2,2, \Omega} \sqrt{\frac{\lambda|\Omega|}{\mu c\left(\hat{F}_{p}\right)}}\left(1+\left\|F_{p}^{-1}\right\|_{2,2, \Omega}\|g\|_{3,2, \Omega}\right)\right.\right. \\
& \left.\left.+\left\|F_{p}^{-1}\right\|_{2,2, \Omega}\left(\|f\|_{2, \Omega}+\|g\|_{2, \Omega}\right)\right]\right) \cdot\left[\left\|F_{p}^{-1}\right\|_{2,2, \Omega}+\left\|\bar{R}_{e}\right\|_{2,2, \Omega}+\|g\|_{3,2, \Omega}+\|f\|_{2, \Omega}\right],
\end{aligned}
$$

implying the boundedness of the minimizing sequence $\left\{\varphi_{n}\right\}_{n=1}^{\infty}$. We may extract a subsequence that converges weakly to some $\varphi \in H^{1,2}(\Omega)$. Since $I$ is convex, it is also sequentially weakly lower semicontinuous which in turn implies

$$
I\left(\varphi, F_{p}^{-1}, \bar{R}_{e}\right) \leq \liminf _{n \rightarrow \infty} I\left(\varphi_{n}, F_{p}^{-1}, \bar{R}_{e}\right)=\inf _{\varphi \in g+H_{\circ}^{1,2}(\Omega)} I\left(\varphi, F_{p}^{-1}, \bar{R}_{e}\right) .
$$

Hence $\varphi$ is a minimizer. Observe that estimate (3.13) remains valid for $\varphi$ by weak lower semicontinuity of the norm.

Theorem 3.3 (Uniqueness of minimizers). Let $F_{p}, F_{p}^{-1} \in H^{2,2}(\Omega, \mathrm{GL}(3, \mathbb{R}))$ and $\bar{R}_{e} \in$ $H^{2,2}(\Omega, \mathrm{SO}(3))$ be given. Assume for the Dirichlet boundary data $g \in H^{3,2}(\Omega)$ and for the body force $f \in L^{2}(\Omega)$. Then the variational problem (3.1) has a unique solution $\varphi \in H^{1,2}(\Omega)$.

Proof. We show that $I$ is strictly convex over the affine space $\left\{g+H_{\circ}^{1,2}(\Omega)\right\}$. This is done by computing the second derivative. We have

$$
\begin{aligned}
& D_{\varphi}^{2} I\left(\varphi, F_{p}^{-1}, \bar{R}_{e}\right) \cdot(\phi, \phi)=\int_{\Omega} \frac{\mu}{2}\left\|F_{p}^{-T} \nabla \phi^{T} \bar{R}_{e}+\bar{R}_{e}^{T} \nabla \phi F_{p}^{-1}\right\|^{2}+\lambda \operatorname{tr}\left[\bar{R}_{e}^{T} \nabla \phi F_{p}^{-1}\right]^{2} \mathrm{dV} \\
& \geq \int_{\Omega} \frac{\mu}{2}\left\|F_{p}^{-T} \nabla \phi^{T} \bar{R}_{e}+\bar{R}_{e}^{T} \nabla \phi F_{p}^{-1}\right\|^{2} \mathrm{dV} \geq \mu c^{+}\left(F_{p}, \bar{R}_{e}, \Omega\right)\|\phi\|_{1,2, \Omega}^{2},
\end{aligned}
$$

by applying Theorem 6.2. Since the Lamé constant $\mu$ is assumed to be strictly positive, we see that $D_{\varphi}^{2} I\left(\varphi, F_{p}^{-1}, \bar{R}_{e}\right) \cdot(\phi, \phi)$ is uniformly positive. Hence $I\left(\varphi, F_{p}^{-1}, \bar{R}_{e}\right)$ is strictly convex.

Corollary 3.4 (General linear system). Let $F_{p}, F_{p}^{-1} \in H^{2,2}\left(\Omega, \mathrm{GL}(3, \mathbb{R})\right.$ ) and $\bar{R}_{e} \in$ $H^{2,2}(\Omega, \mathrm{SO}(3))$ be given and set $A=\left(F_{p}^{-T}, \bar{R}_{e}\right)$. Suppose that $\mathbb{D}$ has the form postulated in Definition 2.4 and assume for the Dirichlet boundary data $g \in H^{3,2}(\Omega)$ and for the right-hand side $f \in L^{2}(\Omega)$. Then the linear problem

$$
\operatorname{Div} \mathbb{D}(A) . \nabla u=f, \quad u_{\left.\right|_{\partial \Omega}}=g,
$$

has a unique weak solution $u \in H^{1,2}(\Omega)$.

Proof. The same ideas as in Theorem 3.2 and Theorem 3.3 carry over. As a corresponding energy expression we have only to take $W_{\mathbb{D}}(F, A)=\frac{\mu}{4}\left\|F_{e}^{T} \bar{R}_{e}+\bar{R}_{e}^{T} F_{e}\right\|^{2}+$ $\frac{\lambda}{8} \operatorname{tr}\left[F_{e}^{T} \bar{R}_{e}+\bar{R}_{e}^{T} F_{e}\right]^{2}$. This result is true for a general right-hand side $f$ and is not necessarily restricted to the body force $f$ appearing in (2.13). 
Theorem 3.5 (Uniform constant in Korn's first inequality). Let $\Omega \subset \mathbb{R}^{3}$ be a bounded domain and let $\Gamma \subset \partial \Omega$ be a part of the boundary with nonvanishing 2-dimensional Hausdorff measure. For given constants $K_{1}, K_{2}>0$, set

$$
\mathcal{M}=\left\{F_{p} \in H^{2,2}(\Omega, G L(3, \mathbb{R})) \mid\left\|F_{p}\right\|_{2,2, \Omega} \leq K_{1},\left\|F_{p}^{-1}\right\|_{2,2, \Omega} \leq K_{2}\right\} .
$$

Then $\exists c_{\mathcal{M}}^{+}>0$ such that $\forall F_{p} \in \mathcal{M} \forall \phi \in H_{\circ}^{1,2}(\Omega, \Gamma)$ :

$$
\left\|\nabla \phi F_{p}^{-1}(x)+F_{p}^{-T}(x) \nabla \phi^{T}\right\|_{L^{2}(\Omega)}^{2} \geq c_{\mathcal{M}}^{+}\|\phi\|_{H^{1,2}(\Omega)}^{2} .
$$

Proof. We proceed by contradiction. Assume without loss of generality that there exists a sequence $\phi_{n} \in H_{\circ}^{1,2}(\Omega, \Gamma):\left\|\phi_{n}\right\|_{1,2, \Omega}=1$ and a sequence $F_{p}^{n} \in \mathcal{M}$ such that

$$
\left\|\nabla \phi_{n} F_{p}^{n,-1}(x)+F_{p}^{n,-T}(x) \nabla \phi_{n}^{T}\right\|_{L^{2}(\Omega)}^{2} \leq \frac{1}{n}\|\phi\|_{H^{1,2}(\Omega)}^{2}=\frac{1}{n} .
$$

Since $F_{p}^{-1, n}$ is bounded in $H^{2,2}(\Omega)$, we may extract a subsequence which converges strongly to some $\hat{F}_{p}^{-1}$ in the topology of $H^{2-\varepsilon, 2}(\Omega)$. Note that the limit $\hat{F}_{p}^{-1} \in \mathcal{M}$ by weak convergence. It is readily seen by continuity and the boundedness of $\phi_{n}$ that this implies on $\mathcal{M}$

$$
\left\|\nabla \phi_{n} \hat{F}_{p}^{-1}(x)+\hat{F}_{p}^{-T}(x) \nabla \phi_{n}^{T}\right\|_{L^{2}(\Omega)}^{2} \rightarrow 0 .
$$

This in turn implies that $\phi_{n}$ is a minimizing sequence. For fixed $\hat{F}_{p}^{-1}$ the quadratic expression is strictly convex in $\phi_{n}$ using Theorem 6.2 in the improved version of 63. We infer that

$$
\left\|\nabla \phi_{n} \hat{F}_{p}^{-1}(x)+\hat{F}_{p}^{-T}(x) \nabla \phi_{n}^{T}\right\|_{L^{2}(\Omega)}^{2} \geq c^{+}\left(\hat{F}_{p}\right)\left\|\phi_{n}\right\|_{H^{1,2}(\Omega)}^{2} .
$$

Hence $\left\|\phi_{n}\right\|_{H^{1,2}(\Omega)}^{2} \leq \frac{1}{c^{+}\left(\hat{F}_{p}\right)} \cdot \frac{1}{n} \rightarrow 0, \quad n \rightarrow \infty$, contradicting $\left\|\phi_{n}\right\|_{H^{1,2}(\Omega)}=1$.

COROLlary 3.6 (Uniform Gårding-type estimate). Under the same assumptions and notation as in Theorem 3.5. let $g \in H^{3,2}(\Omega)$ and $f \in L^{2}(\Omega)$. Then the variational problem (3.1) has a unique solution $\varphi \in H^{1,2}(\Omega)$. For this solution the following estimate is valid:

$$
\begin{aligned}
& \exists C_{\mathcal{M}}^{+}\left(\|g\|_{3,2, \Omega},\|f\|_{2, \Omega}\right)>0 \quad \forall F_{p}, \bar{R}_{e} \in \mathcal{M} \\
& \quad\|\varphi\|_{1,2, \Omega} \leq C_{\mathcal{M}}^{+}\left(\|g\|_{3,2, \Omega},\|f\|_{2, \Omega}\right) \cdot\left(\left\|F_{p}^{-1}\right\|_{2,2, \Omega}+\left\|\bar{R}_{e}\right\|_{2,2, \Omega}+\|g\|_{3,2, \Omega}+\|f\|_{2, \Omega}\right)
\end{aligned}
$$

and $C_{\mathcal{M}}^{+}\left(\|g\|_{3,2, \Omega},\|f\|_{2, \Omega}\right)$ is a continuous function of $\|g\|_{3,2, \Omega}$ and $\|f\|_{2, \Omega}$.

Proof. We recall the estimates of Theorem 3.2. With the assumptions on the coefficients $A$ we have with Theorem 3.5 that the appearing constants in Theorem 3.2 are bounded independent of the coefficients on $\mathcal{M}$. Analyzing estimate (3.13) shows that all appearing constants are uniform on $\mathcal{M}$. Hence the bound is itself uniform.

Lemma 3.7 (Uniform ellipticity). Let $\mathcal{M}$ as in Theorem 3.5 Then $\exists c_{\mathcal{M}}^{+}>0 \quad \forall F_{p} \in$ $\mathcal{M} \forall \xi, \eta \in \mathbb{R}^{3}$ :

$$
\left\|(\xi \otimes \eta) \cdot F_{p}^{-1}(x)+F_{p}^{-T}(x) \cdot(\xi \otimes \eta)^{T}\right\|^{2} \geq c_{\mathcal{M}}^{+} \cdot\|\xi\|^{2}\|\eta\|^{2} .
$$


Proof. A simple algebraic computation shows that

$$
\left\|(\xi \otimes \eta) \cdot F_{p}^{-1}(x)+F_{p}^{-T}(x) \cdot(\xi \otimes \eta)^{T}\right\|^{2} \geq 2 \lambda_{\min }^{2}\left(F_{p}^{-1}(x) F_{p}^{-T}(x)\right) \cdot\|\xi\|^{2}\|\eta\|^{2} .
$$

It is easily verified that there exists a $d_{+}>0$ such that $\operatorname{det}\left[F_{p}^{-1}\right](x) \geq d_{+}$for all $F_{p} \in$ $\mathcal{M}$. Since $d_{+}^{2} \leq \operatorname{det}\left[F_{p}^{-1}(x) F_{p}^{-1}(x)\right]=\lambda_{\min } \cdot \lambda_{2} \cdot \lambda_{\max }\left(F_{p}^{-1}(x) F_{p}^{-T}(x)\right)$, it follows that $\lambda_{\min }^{2} \geq \frac{d_{+}^{4}}{\lambda_{\max }^{4}}$ which in turn implies $\lambda_{\min }^{2} \geq \frac{d_{+}^{4}}{\left\|F_{p}^{-1} F_{p}^{-T}\right\|^{4}}$. Therefore we have a lower bound for the smallest eigenvalue $\lambda_{\min }\left(F_{p}^{-1}(x) F_{p}^{-T}(x)\right)$ on $\mathcal{M}$.

Corollary 3.8 (Uniform ellipticity of the equilibrium system). Set $\hat{F}_{p}=\bar{R}_{e} F_{p}$. Again let $\mathcal{M}$ be defined as in Theorem 3.5. Assume that $F_{p}, \bar{R}_{e} \in \mathcal{M}$. Then the system (2.13) with elasticity tensor $\mathbb{D}$ given by Definition 2.4 is uniformly Legendre-Hadamard elliptic on $\mathcal{M}$,

$$
\exists c_{e, \mathcal{M}}^{+}>0: \forall x \in \Omega: \forall A \in \mathcal{M}: \quad\langle\mathbb{D}(A(x)) .(\xi \otimes \eta), \xi \otimes \eta\rangle \geq c_{e, \mathcal{M}}^{+}\|\xi\|^{2}\|\eta\|^{2}
$$

and the ellipticity constant is given by $c_{e, \mathcal{M}}^{+}=\mu \cdot \lambda_{\min }^{2}\left(\hat{F}_{p}^{-1}(x) \hat{F}_{p}^{-T}(x)\right)$ which is bounded below on $\mathcal{M}$.

Proof. Take $H=\xi \otimes \eta$. We have to compute $D_{F}^{2} W\left(F, F_{p}, \bar{R}_{e}\right) .(H, H)$ with $W$ as in (3.1). It holds true that $D_{F}^{2} W\left(F, F_{p}, \bar{R}_{e}\right) \cdot(H, H) \geq \frac{\mu}{2}\left\|F_{p}^{-T} H^{T} \bar{R}_{e}+\bar{R}_{e}^{T} H F_{p}^{-1}\right\|^{2}$. The relevant identity is

$$
\begin{aligned}
& \left\|F_{p}^{-T} H^{T} \bar{R}_{e}+\bar{R}_{e}^{T} H F_{p}^{-1}\right\|^{2}=\left\|\left(\bar{R}_{e}^{T} \bar{R}_{e}\right) F_{p}^{-T} H^{T} \bar{R}_{e}+\bar{R}_{e}^{T} H F_{p}^{-1}\left(\bar{R}_{e}^{T} \bar{R}_{e}\right)\right\|^{2} \\
& =\left\|\bar{R}_{e}^{T}\left(\bar{R}_{e} F_{p}^{-T} H^{T}+H F_{p}^{-1} \bar{R}_{e}^{T}\right) \bar{R}_{e}\right\|^{2}=\left\|\bar{R}_{e} F_{p}^{-T} H^{T}+H F_{p}^{-1} \bar{R}_{e}^{T}\right\|^{2} \\
& =\left\|\hat{F}_{p}^{-T} H^{T}+H \hat{F}_{p}^{-1}\right\|^{2} .
\end{aligned}
$$

An application of Lemma 3.7 finishes the proof.

Hence with this choice of $\mathbb{D}$ the assumption (GA.4) is fulfilled.

3.3. The visco-plastic evolution problem. In this final part of the proof we consider the coupled visco-elastic-visco-plastic evolution problem. We can write the evolution part of (3) in the following block diagonal form with $A=\left(F_{p}^{-T}, \bar{R}_{e}\right) \in \mathbb{M}^{3 \times 3} \times \mathbb{M}^{3 \times 3}$ :

$$
\frac{\mathrm{d}}{\mathrm{dt}}\left(\begin{array}{c}
F_{p}^{-T}(t) \\
R_{e}(t)
\end{array}\right)=\left(\begin{array}{cc}
-\partial \chi\left(\Sigma_{E}(t)\right)^{T} & 0 \\
0 & \nu^{+} \cdot \operatorname{skew}(B(t))
\end{array}\right) \cdot\left(\begin{array}{c}
F_{p}^{-T}(t) \\
R_{e}(t)
\end{array}\right) .
$$

Thus problem ( $\underline{\mathrm{P} 3})$ is equivalent to

$$
\frac{\mathrm{d}}{\mathrm{dt}} A(t)=\mathfrak{h}\left(\nabla_{x} T(A(t), g(t), f(t)), A(t)\right) \cdot A(t),
$$

with $\mathfrak{h}: \mathbb{M}^{3 \times 3} \times\left(\mathbb{M}^{3 \times 3} \times \mathbb{M}^{3 \times 3}\right) \mapsto \operatorname{Lin}\left(\mathbb{M}^{3 \times 3}, \mathbb{M}^{3 \times 3}\right)=\mathbb{M}^{6 \times 6}$,

$$
\mathfrak{h}(F, A(t))=\left(\begin{array}{cc}
-\partial \chi\left(\Sigma_{M}(t)\right)^{T} & 0 \\
0 & \nu^{+} \cdot \operatorname{skew}(B(t))
\end{array}\right),
$$

where $\Sigma_{E}$ and $B$ are expressions depending on $A=\left(F_{p}^{-T}, \bar{R}_{e}\right)$ and on $F=\nabla \varphi=$ $\nabla_{x} T(A, g, f)$, where $T(A, g, f)$ is formally defined to be the solution operator of the equilibrium part (P3.1) of (P3). In order to account for von Mises type $J_{2}$-visco-plasticity with elastic domain $\mathcal{E}:=\left\{\Sigma_{E} \mid\left\|\operatorname{dev}\left(\operatorname{sym} \Sigma_{E}\right)\right\| \leq \sigma_{\mathrm{y}}\right\}$ and yield stress $\sigma_{\mathrm{y}}$, we take as 
visco-plastic potential

$\chi: \mathbb{M}^{3 \times 3} \mapsto \mathbb{R}$ of generalized Norton-Hoff overstress type the following function:

$$
\chi\left(\Sigma_{E}\right)= \begin{cases}0 & \Sigma_{E} \in \mathcal{E} \\ \frac{\bar{\sigma}_{0}}{(r+1)(k+1) \eta_{p}}\left(1+\left[\frac{\left\|\operatorname{dev}\left(\operatorname{sym} \Sigma_{E}\right)\right\|-\sigma_{\mathrm{y}}}{\bar{\sigma}_{0}}\right]^{r+1}\right)^{k+1}-\frac{\bar{\sigma}_{0}}{(r+1)(k+1) \eta_{p}} & \Sigma_{E} \notin \mathcal{E},\end{cases}
$$

where $\eta_{p}>0$ is the relaxation time of the system due to essentially plastic processes inside the grains and parameters $r, k>0$ and $\bar{\sigma}_{0}$ is a stress-like material constant. An easy calculation shows that this leads to the single-valued subdifferential

$$
\begin{aligned}
& \partial_{\Sigma} \chi\left(\Sigma_{E}\right)=\frac{1}{\eta_{p}} \cdot\left(1+\left[\frac{\left\|\operatorname{dev} \operatorname{sym} \Sigma_{E}\right\|-\sigma_{\mathrm{y}}}{\overline{\sigma_{0}}}\right]_{+}^{r+1}\right)^{k} \\
& \cdot\left[\frac{\left\|\operatorname{dev} \operatorname{sym} \Sigma_{E}\right\|-\sigma_{\mathrm{y}}}{\overline{\sigma_{0}}}\right]_{+}^{r} \frac{\operatorname{dev} \operatorname{sym} \Sigma_{E}}{\left\|\operatorname{dev} \operatorname{sym} \Sigma_{E}\right\|} .
\end{aligned}
$$

The parameter $r$ allows us to adjust the smoothness of the flow rule when passing the elastic boundary. With $r>3$ it is clear that $\partial \chi \in C^{3}\left(\mathbb{M}^{3 \times 3}, \mathbb{M}^{3 \times 3}\right)$. For $k \rightarrow \infty$ we recover formally ideal rate-independent plasticity. Without loss of generality we choose the positive function $\nu^{+}$in the elastic flow part formally similar to (3.31)

$$
\nu^{+}=\frac{1}{\eta_{e}}\left(1+\left[\frac{\left\|\operatorname{skew}\left(\mu F_{e} \bar{R}_{e}^{T}\right)\right\|-0}{\bar{\sigma}_{0}}\right]_{+}^{r+1}\right)^{k} \cdot\left[\frac{\| \text { skew }(B) \|-0}{\bar{\sigma}_{0}}\right]_{+}^{r} \cdot \frac{1}{\| \text { skew }(B) \|} .
$$

Here $\eta_{e}>0$ is the relaxation time of the system due to grain boundary relaxation, which, in view of the results obtained in the companion paper [57] can be assumed to be of the order of $0.01[\mathrm{sec}]$ for polycrystalline metals. This choice makes the flow rule altogether a $C^{3}$-function and implies that $\mathfrak{h} \in C^{3}\left(\mathbb{M}^{3 \times 3} \times\left(\mathbb{M}^{3 \times 3} \times \mathbb{M}^{3 \times 3}\right), \operatorname{Lin}\left(\mathbb{M}^{3 \times 3}, \mathbb{M}^{3 \times 3}\right)\right)$, considered pointwise.

REmark 3.9 (Flow rule on Sobolev space). Set $M:=\left\{v \in H^{m+1,2}(\Omega) \mid\|v\|_{m+1,2, \Omega} \leq\right.$ $K\}$. Then due to Sobolev's embedding theorem it is easy to see that for $\mathfrak{h} \in C^{m+2}\left(\mathbb{M}^{3 \times 3} \times\right.$ $\left.\left(\mathbb{M}^{3 \times 3} \times \mathbb{M}^{3 \times 3}\right), \mathbb{M}^{6 \times 6}\right)$ and $\forall v_{1}, v_{2} \in M$ the estimate

$$
\left\|\mathfrak{h}\left(v_{1}\right)-\mathfrak{h}\left(v_{2}\right)\right\|_{m+1,2, \Omega} \leq \sup _{\|\xi\| \leq \tilde{K}}\|\mathfrak{h}(\xi)\|_{C^{m+2}\left(\mathbb{R}^{27}, \mathbb{M}^{6 \times 6}\right)} \cdot C^{+}(\Omega, M) \cdot\left\|v_{1}-v_{2}\right\|_{m+1,2, \Omega}
$$

holds.

It remains to identify the precise spaces on which to consider this evolution problem in the framework of Theorem 6.1 We let

$$
U:=H^{2,2}(\Omega, \operatorname{GL}(3, \mathbb{R})), \quad X:=H^{2,2}(\Omega, \mathrm{SL}(3, \mathbb{R})) \times H^{2,2}(\Omega, \mathrm{SO}(3))
$$

and we set $Y:=H^{3,2}\left(\Omega, \mathbb{R}^{3}\right)$ and $Z:=H^{1,2}\left(\Omega, \mathbb{R}^{3}\right)$. Assume that $A^{0}=\left(F_{p}^{-T, 0}, \bar{R}_{e}^{0}\right) \in X$ is given with $\operatorname{det}\left[F_{p}^{0}(x)\right]=1$ and for given constants $K_{1}, K_{2}, K_{3}>0$, let

$$
\begin{aligned}
\mathcal{M} & :=\left\{A \in X \mid\left\|A-A^{0}\right\|_{2,2, \Omega} \leq K_{1}\right\}, \quad \mathcal{Y}:=\left\{y \in Y \mid\|y\|_{3,2, \Omega} \leq K_{2}\right\}, \\
\mathcal{Z} & :=\left\{z \in Z \mid\|z\|_{1,2, \Omega} \leq K_{3}\right\} .
\end{aligned}
$$


Observe that by construction of the flow rule (3.27)

$$
\frac{\mathrm{d}}{\mathrm{dt}}\left[F_{p}^{-1}\right](t)=F_{p}^{-1}(t) \cdot \mathfrak{X}_{1}, \quad \frac{\mathrm{d}}{\mathrm{dt}} \bar{R}_{e}(t)=\mathfrak{X}_{2} \cdot \bar{R}_{e}(t),
$$

and since $\mathfrak{X}_{1} \in \mathfrak{s l}(3)$ and $\mathfrak{X}_{2} \in \mathfrak{s o}(3)$, we know a priori that

$$
\operatorname{det}\left[F_{p}(x, t)\right]=1, F_{p}(x, t) \in \mathrm{SL}(3) \quad \text { and } \quad \bar{R}_{e}(x, t) \in \mathrm{SO}(3) .
$$

We assume for the Dirichlet boundary data $g \in C^{1}([0, T], \mathcal{Y})$ and for the body forces $f \in C^{1}([0, T], \mathcal{Z})$. In view of the above statements we show presently that the evolution problem

$$
\frac{\mathrm{d}}{\mathrm{dt}} A(t)=\mathfrak{h}\left(\nabla_{x} T(A(t), g(t), f(t)), A(t)\right) \cdot A(t)
$$

fits into the formal framework set forth in Theorem 6.1

First we proceed to show it is possible to define a solution operator $\varphi=T\left(F_{p}, \bar{R}_{e}, g, f\right)$ in the static equilibrium part ( (P3.1) of (P3) and that this operator is indeed Lipschitz continuous on $\mathcal{M} \times \mathcal{Y} \times \mathcal{Z}$.

Due to Theorem 3.2 and Lemma 2.3 we know that solutions $\varphi$ of (P3.1) exist. With Definition 2.4 it is clear that $\mathbb{D}, V \in C^{\infty}$. Remark 3.9 shows that (GA.3) and (GA.5) are verified for $\mathbb{D}, V$ on $\mathcal{M}$. Moreover, by Lemma 3.7] we see that (GA.4) is true. If we choose the order of elliptic regularity $m=1$ for the space dimension $n=3$, then (GA.2) holds as well. Of course we have to assume (GA.1).

Theorem 3.3 shows that the solutions of the boundary value problem (P3.1) are unique, which establishes existence of the solution operator and Corollary 3.6 proves a uniform $H^{1,2}(\Omega)$ estimate for $g \in \mathcal{Y}, f \in \mathcal{Z}$ on $\mathcal{M}$. With Corollary 3.4 we make sure that the assumptions of Theorem 6.5 are verified. Therefore we are entitled to apply elliptic regularity; cf. Theorem 6.6 and Corollary 6.7. Altogether, this yields that $T(A, g, f)$ is a continuous operator with uniform Lipschitz constant $L^{+}$on $\mathcal{M} \times \mathcal{Y} \times \mathcal{Z}$. The gradient $\nabla_{x} T(A, g, f)$ satisfies the same type of uniform Lipschitz estimate, namely

$$
\begin{aligned}
\| \nabla_{x} T & \left(A, g_{A}, f_{A}\right)-\nabla_{x} T\left(B, g_{B}, f_{B}\right) \|_{m+1,2, \Omega} \\
\leq & C^{+}(\Omega, \mathcal{M}) \cdot\left(1+\|B\|_{m+1,2, \Omega}+\left\|g_{B}\right\|_{m+2,2, \Omega}+\left\|f_{B}\right\|_{m+2,2, \Omega}\right) \\
& \times\left(\|A-B\|_{m+1,2, \Omega}+\left\|g_{A}-g_{B}\right\|_{m+2,2, \Omega}+\left\|f_{A}-f_{B}\right\|_{m, 2, \Omega}\right) .
\end{aligned}
$$

Hence on $\mathcal{M} \times \mathcal{Y} \times \mathcal{Z}$ we obtain

$$
\begin{aligned}
& \| \nabla_{x} T\left(A, g_{A}, f_{A}\right)-\nabla_{x} T\left(B, g_{B}, f_{B}\right) \|_{m+1,2, \Omega} \\
& \leq C^{+}(\Omega, \mathcal{M}) \cdot\left(1+K_{1}+K_{3}\right) \\
& \quad \times\left(\|A-B\|_{m+1,2, \Omega}+\left\|g_{A}-g_{B}\right\|_{m+2,2, \Omega}+\left\|f_{A}-f_{B}\right\|_{m, 2, \Omega}\right) .
\end{aligned}
$$

This is enough to see that the operator $G(A, g, f):=\nabla_{x} T(A, g, f)$ verifies the condition of Theorem 6.1

Moreover, Remark 3.9 applied to $\mathfrak{h} \in C^{3}\left(\mathbb{M}^{3 \times 3} \times \mathbb{M}^{3 \times 3}, \mathbb{M}^{6 \times 6}\right)$ shows that $\mathfrak{h}$, viewed as a function $\mathfrak{h}: U \times X \mapsto \operatorname{Lin}(X, X)$, is locally Lipschitz continuous on $\mathcal{M}$. Therefore, we may finally apply Theorem 6.1 giving us a unique local in time solution $A \in C^{1}\left(\left[0, t_{1}\right], \mathcal{M}\right)$ to the ordinary differential system of equations (3.28). Since $\varphi(t)=T(A(t), g(t), f(t))$, 
the pair $(\varphi, A) \in C\left(\left[0, t_{1}\right], H^{3,2}\left(\Omega, \mathbb{R}^{3}\right)\right) \times C^{1}\left(\left[0, t_{1}\right], \mathcal{M}\right)$ is the unique local in time solution of (푸).

This finishes the proof of Theorem 2.1

4. Discussion and concluding remarks. Having proved a local existence theorem for the viscoelastic-viscoplastic case of ( $(\mathrm{P} 3)$ we observe that the existence time in general will depend crucially on the smoothness of the values $\left(F_{p}, \bar{R}_{e}\right)$, i.e., the smoothness of the elasticity tensor $\mathbb{D}$. If bifurcations occur, they must then be attributed to a severe loss of smoothness of these elastic moduli.

It is still an open problem whether the viscoelastic-viscoplastic system (P3) admits global in time solutions for small data. This may not be true since the loss of smoothness might accumulate with time even for small and smooth given data, e.g., under sustained low amplitude cyclic loading, therefore allowing for fatigue phenomena. The smoothness assumptions made in Theorem 2.1 reflect accurately the possible macroscopic elastic failure of the polycrystalline material. Since in a Sobolev-space context we need at least $F_{p} \in H^{1,3+\delta}\left(\Omega, \mathrm{GL}^{+}(3, \mathbb{R})\right)$, in order to guarantee the minimal constitutive requirement $F_{p} \in L^{\infty}\left(\Omega, \mathrm{GL}^{+}(3, \mathbb{R})\right)$, the regularity gap $F_{p} \in H^{2,2}\left(\Omega, \mathrm{GL}^{+}(3, \mathbb{R})\right) \subset$ $H^{1,3+\delta}\left(\Omega, \mathrm{GL}^{+}(3, \mathbb{R})\right)$ is small and the result is practically optimal.

I presume that the situation is completely different for the rate-independent case; here it seems that the peculiar form of the flow rule is responsible for elastic-plastic instabilities. The foregoing analysis, however, is strictly confined to the rate-dependent case, but, contrary to the variational approach, not restricted to associated plasticity.

In the rate-independent problem following [59] for the single crystal case it is believed that one can ascribe observed microstructure inside one crystal grain (weak discontinuities or shear bands, i.e., a jump of the deformation gradient and strong discontinuities or slip lines, i.e., a jump of the deformation itself) to the lack of quasiconvexity of an associated incremental potential which derives from a fully implicit time discretization of (P0) in the quasistatic case. Based on this observation a promising new approach has been taken towards a numerical and mathematical analysis; see [26, 10, 48, 60, 61]. The lack of quasiconvexity of the incrmental problem is, however, not at variance with the assumed ellipticity of the purely elastic problem. It is conceivable that the time-incremental problem derived from $(\mathbb{P 3})$ is not quasiconvex (even in the viscous case with smooth initial elastic moduli) leading to the tentative conclusion that the local solutions found in Theorem 2.1 do not necessarily realize global minima of the incremental potential but rather stable local minima and that viscosity prevents the formation of microstructure in the small elastic strain regime on the macroscopic scale. The above-mentioned discontinuities of the deformation inside the grains are therefore not at variance with the general smoothness level required in Theorem 2.1, since, e.g., $F_{p}$ in (P3) corresponds conceptually to the averaged plastic 'deformation' of the grains while $\bar{R}_{e}$ is a suitable average of rotations.

In closing, a number of possible extensions of the theory are worth mentioning. The general mathematical methodology of (P3) is not confined to a polycrystal setting. In the case of single crystal visco-plasticity one simply has to exchange the flow rule for $F_{p}$ and one can expect the same type of results. Likewise isotropic and kinematic hardening 
can be incorporated without difficulty. The use of unified constitutive equations [66] without elastic domain is also possible as well as a nonassociated formulation provided that the flow rule is locally Lipschitz continuous.

First numerical computations [58] with the relaxation time $\eta_{e}$ of the order 0.01 and $B=B_{\text {mech }}$ confirm the general applicability of the model ( $(\mathrm{P} 3)$ for structural applications compared with standard models and corroborate the excellent properties of (플 choice in the evolution of the 'visco-elastic' rotations without yet exploring the specific texture effects inherent in (P3).

5. Acknowledgements. The author would like to express his gratitude to K. Chelminski and S. Ebenfeld for helpful discussions. This article was conceived in late 2000 [55]. The author acknowledges the helpful remarks of various reviewers.

\section{Appendix.}

6.1. Ordinary differential equations in Banach spaces. The following result is a simple consequence of Banach's fixed-point theorem.

Theorem 6.1 (Unique local existence). Let $U, X, Y, Z$ be arbitrary Banach spaces with norms $\|\cdot\|_{U},\|\cdot\|_{X},\|\cdot\|_{Y},\|\cdot\|_{Z}$, respectively. Assume that $h: U \times X \mapsto \operatorname{Lin}(X, X)$ is locally Lipschitz continuous and let the initial value $y^{0} \in X$ be given. Let $G: X \times Y \times Z \mapsto U$ be an operator which is Lipschitz continuous on the set $\mathcal{M} \times \mathcal{Y} \times \mathcal{Z}$ with $\mathcal{M}:=\{y \in$ $\left.X \mid\left\|y-y^{0}\right\|_{X} \leq K\right\}$ and $\mathcal{Y} \subset Y, \mathcal{Z} \subset Z$ bounded in $Y, Z$, respectively, i.e., there is a positive constant $L^{+}$such that

$$
\begin{aligned}
& \exists L^{+}>0: \quad \forall\left(x_{1}, a_{1}, b_{1}\right),\left(x_{2}, a_{2}, b_{2}\right) \in \mathcal{M} \times \mathcal{Y} \times \mathcal{Z}: \\
& \left\|G\left(x_{1}, a_{1}, b_{1}\right)-G\left(x_{2}, a_{2}, b_{2}\right)\right\|_{U} \leq L^{+} .\left(\left\|x_{1}-x_{2}\right\|_{X}+\left\|a_{1}-a_{2}\right\|_{Y}+\left\|b_{1}-b_{2}\right\|_{Z}\right) .
\end{aligned}
$$

Moreover, assume that $\alpha \in C^{1}([0, T], \mathcal{Y}), \beta \in C^{1}([0, T], \mathcal{Z})$ are given functions. Then there is some $0<t_{1} \in \mathbb{R}$ such that the initial value problem

$$
\frac{\mathrm{d}}{\mathrm{dt}} y(t)=h(G(y(t), \alpha(t), \beta(t)), y(t)) \cdot y(t), \quad y(0)=y^{0},
$$

has a unique solution $y \in C^{1}\left(\left[0, t_{1}\right], \mathcal{M}\right)$.

\subsection{Extended Korn first inequality.}

Theorem 6.2 (Extended 3D Korn first inequality). Let $\Omega \subset \mathbb{R}^{3}$ be a bounded Lipschitz domain and let $\Gamma \subset \partial \Omega$ be a smooth part of the boundary with nonvanishing 2-dimensional Lebesgue measure. Define $H_{\circ}^{1,2}(\Omega, \Gamma):=\left\{\phi \in H^{1,2}(\Omega) \mid \phi_{\left.\right|_{\Gamma}}=0\right\}$ and let $F_{p}, F_{p}^{-1} \in C^{1}(\bar{\Omega}, \mathrm{GL}(3, \mathbb{R}))$. Moreover suppose that $\operatorname{Curl} F_{p} \in C^{1}\left(\bar{\Omega}, \mathbb{M}^{3 \times 3}\right)$. Then

$$
\exists c^{+}>0 \forall \phi \in H_{\circ}^{1,2}(\Omega, \Gamma): \quad\left\|\nabla \phi F_{p}^{-1}(x)+F_{p}^{-T}(x) \nabla \phi^{T}\right\|_{L^{2}(\Omega)}^{2} \geq c^{+}\|\phi\|_{H^{1,2}(\Omega)}^{2} .
$$

Proof. The proof has been presented in [56]. Note that for $F_{p}=\nabla \Theta$ we would only have to deal with the classical Korn inequality evaluated on the transformed domain $\Theta(\Omega)$. However, in general, $F_{p}$ is incompatible, giving rise to a non-Riemannian manifold structure. Compare to [13] for an interpretation and the physical relevance of 
the quantity $\operatorname{Curl} F_{p}$. It comes as no surprise that in finite plasticity the incompatibility of $F_{p}$ should play an important role.

Motivated by the investigations in [56], it has been shown recently by Pompe [63] that the extended Korn inequality can be viewed as a special case of a general class of coerciveness inequalities for quadratic forms. He was able to show that indeed $F_{p}, F_{p}^{-1} \in$ $C(\bar{\Omega}, \mathrm{GL}(3, \mathbb{R}))$ is sufficient for Theorem 6.2 to hold without any condition on the compatibility.

However, taking the special structure of the extended Korn inequality into account again, work in progress suggests that continuity is not really necessary: instead $F_{p} \in$ $L^{\infty}(\Omega, \mathrm{GL}(3, \mathbb{R}))$ and $\operatorname{Curl} F_{p} \in L^{3+\delta}(\Omega)$ should suffice, whereas $F_{p} \in L^{\infty}(\Omega, \operatorname{GL}(3, \mathbb{R}))$ alone is not sufficient; see the counterexample presented in [63]. The possible improvement has no bearing on our further development.

6.3. Sharp ellipticity type estimates. Let us gather results that are necessary for the exposition of the static case. We need sharp a priori estimates for elliptic systems of second order with nonconstant coefficients in divergence form. Ebenfeld 22] has recently proved the following new sharpened a priori estimate which we give adapted to our situation and our notation.

Theorem 6.3 (General improved sharp Hilbert space elliptic regularity). Let $\Omega \subset \mathbb{R}^{n}$ be a bounded domain with smooth boundary. Consider the divergence-form linear system

$$
\operatorname{Div} \mathbb{C}(x) . \nabla u=f(x), \quad u_{\mid \partial \Omega}=0,
$$

with $f \in H^{m, 2}(\Omega)$ and homogeneous boundary data. Let $\mathbb{C}: \Omega \subset \mathbb{R}^{3} \mapsto \operatorname{Lin}\left(\mathbb{M}^{3 \times 3}, \mathbb{M}^{3 \times 3}\right)$ be the elasticity tensor. Suppose $\mathbb{C} \in H^{m+1,2}(\Omega)$ with $2 \cdot(m+1)>n$ and assume that for arbitrary $\xi, \eta \in \mathbb{R}^{n}$ it holds that

$$
\exists c_{e}^{+}>0 \quad \forall x \in \Omega: \quad\langle\mathbb{C}(x) \cdot(\xi \otimes \eta), \xi \otimes \eta\rangle \geq c_{e}^{+} \cdot\|\xi\|^{2}\|\eta\|^{2},
$$

i.e., that the system is uniformly Legendre-Hadamard elliptic with ellipticity constant $c_{e}^{+}$. Assume that the system admits at least one weak solution $u \in H^{1,2}(\Omega)$. Then the following estimate is valid:

$$
\|u\|_{m+2,2, \Omega} \leq C^{+}\left(\Omega, c_{e}^{+}\right) P\left(\|\mathbb{C}\|_{m+1,2, \Omega}\right)\left(\|f\|_{m, 2, \Omega}+\|u\|_{2, \Omega}\right),
$$

where $P: \mathbb{R} \mapsto \mathbb{R}$ is a polynomial of finite order and the appearing constant is independent of $u, f, \mathbb{C}$ and in addition $C^{+}\left(\Omega, c_{e}^{+}\right)$is bounded above for $c_{e}^{+}>0$.

Proof. See [20, 21] and compare with [73, p. 75] for comparable results on elliptic regularity for linear second order elliptic systems on other scales. The main advantage of the new theorem is to precisely track how the regularity of the coefficients enter the elliptic estimate. Precise estimates of this form had not been available previously.

Now we specialize the general estimate to our situation.

Theorem 6.4 (Improved Hilbert space elliptic regularity with $L^{2}$-part). Assume GA and $A \in \mathcal{M}$. Consider the linear divergence form elliptic system

$$
\operatorname{Div} \mathbb{D}(A) . \nabla u=f(x), \quad u_{\left.\right|_{\partial \Omega}}=g(x) .
$$


Assume that (6.4) admits at least one weak solution $u \in H^{1,2}(\Omega)$ for all $g \in H^{m+2,2}(\Omega)$ and all $f \in H^{m, 2}(\Omega)$. Then the estimate

$$
\|u\|_{m+2,2, \Omega} \leq C^{+}\left(\Omega,\|\mathbb{D}(A)\|_{m+1,2, \Omega}\right) \cdot\left(\|g\|_{m+2,2, \Omega}+\|f\|_{m, 2, \Omega}+\|u\|_{2, \Omega}\right)
$$

is valid, and the appearing constant $C^{+}\left(\Omega,\|\mathbb{D}(A)\|_{m+1,2, \Omega}\right)$ is uniform on $\mathcal{M}$.

Proof. The transformation $v=u-g$ allows us to consider

$$
\operatorname{Div} \mathbb{D}(A) . \nabla v=f(x)+\operatorname{Div} \mathbb{D}(A) . \nabla g, \quad v_{\mid \partial \Omega}=0 .
$$

If we apply Theorem 6.3 to (6.6), we get the estimate

$$
\begin{aligned}
\|v\|_{m+2,2, \Omega} \leq & C^{+}\left(\Omega, c_{e}^{+}\right) P\left(\|\mathbb{D}(A)\|_{m+1,2, \Omega}\right) \\
& \times\left(\|\operatorname{Div} \mathbb{D}(A) . \nabla g\|_{m, 2, \Omega}+\|f\|_{m, 2, \Omega}+\|v\|_{2, \Omega}\right), \\
\|v\|_{m+2,2, \Omega} \leq & C^{+}\left(\Omega, c_{e}^{+}\right) P\left(\|\mathbb{D}(A)\|_{m+1,2, \Omega}\right) \\
& \times\left(\|\mathbb{D}(A)\|_{m+1,2, \Omega}\|g\|_{m+2,2, \Omega}+\|f\|_{m, 2, \Omega}+\|v\|_{2, \Omega}\right), \\
\|v\|_{m+2,2, \Omega} \leq & C^{+}\left(\Omega, c_{e}^{+}\right) P\left(\|\mathbb{D}(A)\|_{m+1,2, \Omega}\right)\left[1+\|\mathbb{D}(A)\|_{m+1,2, \Omega}\right] \\
& \times\left(\|g\|_{m+2,2, \Omega}+\|f\|_{m, 2, \Omega}+\|u\|_{2, \Omega}+\|g\|_{2, \Omega}\right) .
\end{aligned}
$$

This yields for $u=v+g$

$$
\begin{aligned}
\|u\|_{m+2,2, \Omega} \leq 2 & \left(1+C^{+}\left(\Omega, c_{e}^{+}\right) P\left(\|\mathbb{D}(A)\|_{m+1,2, \Omega}\right)\left[1+\|\mathbb{D}(A)\|_{m+1,2, \Omega}\right]\right) \\
& \times\left(\|g\|_{m+2,2, \Omega}+\|f\|_{m, 2, \Omega}+\|u\|_{2, \Omega}\right) .
\end{aligned}
$$

Now take

$$
C^{+}\left(\Omega,\|\mathbb{D}(A)\|_{m+1,2, \Omega}\right)=2\left(1+C^{+}\left(\Omega, c_{e}^{+}\right) P\left(\|\mathbb{D}(A)\|_{m+1,2, \Omega}\right)\left[1+\|\mathbb{D}(A)\|_{m+1,2, \Omega}\right]\right) .
$$

This ends the proof since $C^{+}\left(\Omega, c_{e}^{+}\right)$is uniformly bounded above on $\mathcal{M}$ by (GA.4) and Theorem 6.3

Theorem 6.5 (Uniform estimates for bounded coefficients). Assume GA and $A \in \mathcal{M}$. Consider the linear divergence form elliptic system

$$
\operatorname{Div} \mathbb{D}(A) . \nabla u=f(x), \quad u_{\left.\right|_{\partial \Omega}}=g(x) .
$$

Assume that (6.9) has a unique weak solution $u \in H^{1,2}(\Omega)$ for all $g \in H^{m+2,2}(\Omega)$ and all $f \in H^{m, 2}(\Omega)$. In addition assume that a uniform Gårding type $L^{2}(\Omega)$-estimate on $\mathcal{M}$ is available, i.e.,

$$
\exists C_{\mathcal{M}}>0: \quad \forall A \in \mathcal{M}: \quad\|u\|_{2, \Omega} \leq C_{\mathcal{M}} \cdot\left(\|g\|_{m_{1}+2,2, \Omega}+\|f\|_{m_{2}, 2, \Omega}\right),
$$

with $\max \left(m_{1}, m_{2}\right) \leq m$. Then the uniform estimate

$$
\|u\|_{m+2,2, \Omega} \leq C^{+}(\Omega, \mathcal{M}) \cdot\left(\|g\|_{m+2,2, \Omega}+\|f\|_{m, 2, \Omega}\right)
$$

is true, and the appearing constant $C^{+}(\Omega, \mathcal{M})$ is uniform on $\mathcal{M}$.

Proof. An application of Theorem 6.4 will give the result. 
Theorem 6.6 (Lipschitz-continuous dependence of solutions). Assume GA and let $A, B \in \mathcal{M}$. Assume for the boundary data $g_{A}, g_{B} \in H^{m+2,2}(\Omega)$ and for the body forces $f_{A}, f_{B} \in H^{m, 2}(\Omega)$. Consider the two systems

Div $\mathbb{D}(A(x)) \cdot \nabla u=f_{A}(x)+\operatorname{Div} V(A) \quad \operatorname{Div} \mathbb{D}(B(x)) \cdot \nabla u=f_{B}(x)+\operatorname{Div} V(B)$,

$$
u_{\left.\right|_{\partial \Omega}}=g_{A}(x) \quad u_{\left.\right|_{\partial \Omega}}=g_{B}(x) .
$$

Assume that both systems verify the assumptions made in Theorem 6.5. Denote the (unique) solutions $u_{A}, u_{B} \in H^{1,2}(\Omega)$, respectively. Then the following estimate holds:

$$
\begin{aligned}
\left\|u_{A}-u_{B}\right\|_{m+2,2, \Omega} \leq C^{+} & (\Omega, \mathcal{M}) \cdot\left(1+\|B\|_{m+1,2, \Omega}+\left\|g_{B}\right\|_{m+2,2, \Omega}+\left\|f_{B}\right\|_{m, 2, \Omega}\right) \\
& \times\left(\|A-B\|_{m+1,2, \Omega}+\left\|g_{A}-g_{B}\right\|_{m+2,2, \Omega}+\left\|f_{A}-f_{B}\right\|_{m, 2, \Omega}\right),
\end{aligned}
$$

with $C^{+}(\Omega, \mathcal{M})$ uniformly bounded on $\mathcal{M}$.

Proof. Consider

$\operatorname{Div} \mathbb{D}(A(x)) . \nabla u=f_{A}(x)+\operatorname{Div} V(A), \quad \operatorname{Div} \mathbb{D}(B(x)) . \nabla u=f_{B}(x)+\operatorname{Div} V(B)$,

$$
u_{\mid \partial \Omega}=g_{A}(x), \quad u_{\mid \partial \Omega}=g_{B}(x) .
$$

Taking the difference of the two equations leads us to consider

$$
\begin{aligned}
\operatorname{Div} \mathbb{D}(A(x)) . \nabla\left(u_{A}-u_{B}\right)= & \operatorname{Div}\left((\mathbb{D}(B(x))-\mathbb{D}(A(x))) \cdot \nabla u_{B}\right) \\
& +f_{A}-f_{B}+\operatorname{Div}(V(A)-V(B)), \\
\left(u_{A}-u_{B}\right)_{\left.\right|_{\partial \Omega}}= & g_{A}-g_{B} .
\end{aligned}
$$

By the assumption on $A$ and the elasticity tensor $\mathbb{D}(A)$ we know that the system (6.15) has a unique solution in the difference $\left(u_{A}-u_{B}\right)$ for this specific right-hand side; see (6.18). Together with the regularity assumption made for $A$ and $\mathbb{D}(A)$ in GA we can apply Theorem 6.5 to (6.15) and get the estimate

$$
\begin{aligned}
\left\|u_{A}-u_{B}\right\|_{m+2,2, \Omega} \leq C^{+}(\Omega, \mathcal{M}) & +\left(\left\|\operatorname{Div}(\mathbb{D}(B)-\mathbb{D}(A)) \cdot \nabla u_{B}\right\|_{m, 2, \Omega}\right. \\
& +\|\operatorname{Div}(V(B)-V(A))\|_{m, 2, \Omega} \\
& \left.+\left\|g_{A}-g_{B}\right\|_{m+2,2, \Omega}+\left\|f_{A}-f_{B}\right\|_{m, 2, \Omega}\right), \\
\left\|u_{A}-u_{B}\right\|_{m+2,2, \Omega} \leq C^{+}(\Omega, \mathcal{M}) \cdot & \left(\|\mathbb{D}(A)-\mathbb{D}(B)\|_{m+1,2, \Omega} \cdot\left\|u_{B}\right\|_{m+2,2, \Omega}\right. \\
& +\|V(B)-V(A)\|_{m+1,2, \Omega} \\
& \left.+\left\|g_{A}-g_{B}\right\|_{m+2,2, \Omega}+\left\|f_{A}-f_{B}\right\|_{m, 2, \Omega}\right) .
\end{aligned}
$$

Again with Theorem 6.5 applied to the solution $u_{B}$, we have

$$
\left\|u_{B}\right\|_{m+2,2, \Omega} \leq C^{+}(\Omega, \mathcal{M}) \cdot\left(\left\|g_{B}\right\|_{m+2,2, \Omega}+\left\|f_{B}\right\|_{m, 2, \Omega}+\|V(B)\|_{m+1,2, \Omega}\right) .
$$

Combining these two estimates and using (GA.5) for $\mathbb{D}, V$ ends the argument. 
COROLlary 6.7 (Lipschitz-continuous solution operator; time dependent coefficients). Assume that for a given family $\mathfrak{M}:=\left\{A_{t} \in \mathcal{M} \mid t>0\right\}$, the family of related elasticity tensors $\mathbb{D}\left(A_{t}\right)$ verifies all conditions of Theorem 6.5 Define

$$
\mathfrak{G}:=\left\{g \in H^{m+2,2}(\Omega) \mid\|g\|_{m+2,2, \Omega} \leq K_{2}\right\}
$$

and

$$
\mathfrak{F}:=\left\{f \in H^{m, 2}(\Omega) \mid\|f\|_{m, 2, \Omega} \leq K_{3}\right\} .
$$

Let the boundary data $g_{t} \in \mathfrak{G}$ and the body forces $f_{t} \in \mathfrak{F}$ be given. Then the family of corresponding linear elliptic systems

$$
\operatorname{Div} \mathbb{D}\left(A_{t}\right) \cdot \nabla \varphi_{t}=f_{t}(x)+\operatorname{Div} V\left(A_{t}\right),\left.\quad \varphi_{t}\right|_{\partial \Omega}=g_{t}(x),
$$

allows for a Lipschitz-continuous solution operator $T$ on $\mathfrak{M} \times \mathfrak{G} \times \mathfrak{F}$ such that $\varphi_{t}=$ $T\left(A_{t}, g_{t}, f_{t}\right)$ and

$$
\begin{aligned}
& \left\|T\left(A, g_{A}, f_{A}\right)-T\left(B, g_{B}, f_{B}\right)\right\|_{m+2,2, \Omega} \\
& \leq C^{+}(\Omega, \mathcal{M}) \cdot\left(1+\|B\|_{m+1,2, \Omega}+\left\|g_{B}\right\|_{m+2,2, \Omega}+\left\|f_{B}\right\|_{m, 2, \Omega}\right) \\
& \times\left(\|A-B\|_{m+1,2, \Omega}+\left\|g_{A}-g_{B}\right\|_{m+2,2, \Omega}+\left\|f_{A}-f_{B}\right\|_{m, 2, \Omega}\right),
\end{aligned}
$$

for $A, B \in \mathfrak{M}, g_{A}, g_{B} \in \mathfrak{G}, f_{A}, f_{B} \in \mathfrak{F}$. The corresponding Lipschitz constant $L^{+}$on $\mathfrak{M} \times \mathfrak{G} \times \mathfrak{F}$ is a bounded function of the form

$$
L^{+}=L^{+}\left(C^{+}(\Omega, \mathcal{M}), K_{2}, K_{3}\right) .
$$

Hence a family of elliptic systems of the above type has corresponding solution operators with uniform Lipschitz constant whenever $\|A\|_{m+1,2, \Omega},\left\|g_{A}\right\|_{m+2,2, \Omega},\left\|f_{A}\right\|_{m, 2, \Omega}$ are bounded due to Theorem6.5.

REMARK 6.8 (Nonlinear solution operator). Let $A_{t} \in \mathcal{M}$ and let $f_{t}, g_{t}$ be as before. Then $\left(f_{t}, g_{t}\right) \mapsto T\left(A_{0}, g_{t}, f_{t}\right)$ is linear and $A_{t} \mapsto T\left(A_{t}, g_{0}, f_{0}\right)$ is nonlinear. Hence the solution depends nonlinearly on the elasticity tensor although the problem is linear for frozen (fixed at time $t_{0}$ ) elasticity tensor $\mathbb{D}\left(A_{t_{0}}\right)$.

\section{REFERENCES}

[1] H.D. Alber, Materials with Memory. Initial-Boundary Value Problems for Constitutive Equations with Internal Variables, Lecture Notes in Mathematics, vol. 1682, Springer, Berlin, 1998. MR.1619546 (99i:73040)

[2] K.H. Anthony, Die Theorie der nichtmetrischen Spannungen in Kristallen, Arch. Rat. Mech. Anal. 40 (1971), 50-78. MR0267823 (42:2725)

[3] A. Bertram, An alternative approach to finite plasticity based on material isomorphisms, Int. J. Plasticity 52 (1998), 353-374.

[4] A. Bertram and B. Svendsen, On material objectivity and reduced constitutive equations, Arch. Mech. 53 (2001), 653-675. MR1885766 (2002m:74003)

[5] J.F. Besseling and E. van der Giessen, Mathematical Modelling of Inelastic Deformation, Applied Mathematics and Mathematical Computation, vol. 5, Chapman Hall, London, 1994. MR1284037 (95j:73044)

[6] K. Bhattacharya and R.V. Kohn, Elastic energy minimization and the recoverable strains of polycrystalline shape memory materials, Arch. Rat. Mech. Anal. 139 (1997), 99-180. MR1478776 $(98 \mathrm{j}: 73025)$ 
[7] F. Bloom, Modern Differential Geometric Techniques in the Theory of Continuous Distributions of Dislocations, Lecture Notes in Mathematics, vol. 733, Springer, Berlin, 1979. MR0546839 (80k:73001)

[8] S.R. Bodner and Y. Partom, Constitutive equations for elastic-viscoplastic strainhardening materials, J. Appl. Mech. 42 (1975), 385-389.

[9] O.T. Bruhns, H. Xiao, and A. Mayers, Some basic issues in traditional eulerian formulations of finite elastoplasticity, Int. J. Plast. 19 (2003), 2007-2026.

[10] C. Carstensen and K. Hackl, On microstructure occurring in a model of finite-strain elastoplasticity involving a single slip system, ZAMM (2000).

[11] C. Carstensen, K. Hackl, and A. Mielke, Non-convex potentials and microstructure in finite strain plasticity, Proc. Roy. Soc. London, Ser. A 458 (2002), 299-317. MR1889770 (2002m:74009)

[12] J. Casey and P.M. Naghdi, A remark on the use of the decomposition $F=F_{e} \cdot F_{p}$ in plasticity, J. Appl. Mech. 47 (1980), 672-675.

[13] P. Cermelli and M.E. Gurtin, On the characterization of geometrically necessary dislocations in finite plasticity, J. Mech. Phys. Solids 49 (2001), 1539-1568.

[14] K. Chelminski, On self-controlling models in the theory of inelastic material behaviour of metals, Cont. Mech. Thermodyn. 10 (1998), 121-133. MR1634932 (99f:73043)

[15] _ On monotone plastic constitutive equations with polynomial growth condition, Math. Meth. App. Sc. 22 (1999), 547-562. MR1682911 (2000b:74018)

[16] P.G. Ciarlet, Three-Dimensional Elasticity, first ed., Studies in Mathematics and its Applications, vol. 1, Elsevier, Amsterdam, 1988. MR0936420 (89e:73001)

[17] Y.F. Dafalias, The plastic spin concept and a simple illustration of its role in finite plastic transformations, Mechanics of Materials 3 (1984), 223-233.

[18] _ Issues on the constitutive formulation at large elastoplastic deformations, Part I: Kinematics, Acta Mechanica 69 (1987), 119-138.

[19] _ Plastic spin: necessity or redundancy?, Int. J. Plasticity 14 (1998), no. 9, 909-931.

[20] S. Ebenfeld, Aspekte der Kontinua mit Mikrostruktur, Berichte aus der Mathematik, Shaker Verlag, Aachen, 1998.

$[21] \_, L^{2}$-regularity theory of linear strongly elliptic Dirichlet systems of order $2 m$ with minimal regularity in the coefficients, Preprint Nr. 2015, TU Darmstadt, (1998).

$[22] \_, L^{2}$-regularity theory of linear strongly elliptic Dirichlet systems of order $2 m$ with minimal regularity in the coefficients, Quart. Appl. Math. 60 (2002), no. 3, 547-576. MR1914441 (2003d:35071)

[23] P. Ellsiepen and S. Hartmann, Remarks on the interpretation of current non-linear finite-elementanalyses as differential-algebraic equations, Int. J. Num. Meth. Engrg. 51 (2001), 679-707.

[24] A.E. Green and P.M. Naghdi, A general theory of an elastic-plastic continuum, Arch. Rat. Mech. Anal. 18 (1965), 251-281. MR0223129|(36:6178)

[25] M.E. Gurtin, On the plasticity of single crystals: free energy, microforces, plastic-strain gradients, J. Mech. Phys. Solids 48 (2000), 989-1036. MR1746552 (2001e:74024)

[26] K. Hackl, Generalized standard media and variational principles in classical and finite strain elastoplasticity, J. Mech. Phys. Solids 45 (1997), 667-688. MR1447372 (97m:73026)

[27] W. Han and B.D. Reddy, Plasticity. Mathematical Theory and Numerical Analysis, Springer, Berlin, 1999. MR:1681061 (2000b:74014)

[28] P. Haupt, On the concept of an intermediate configuration and its application to a representation of viscoelastic-plastic material behaviour, Int. J. Plasticity 1 (1985), 303-316.

[29] - Continuum Mechanics and Theory of Materials, Springer, Heidelberg, 2000. MR1762655 (2001j:74001)

[30] I.R. Ionescu and M. Sofonea, Functional and Numerical Methods in Viscoplasticity, first ed., Oxford Mathematical Monographs, Oxford University Press, Oxford, 1993. MR 1244578 (95e:73025)

[31] L.M. Kachanov, Introduction to Continuum Damage Mechanics, Martinus Nijhoff Publishers, Dordrecht, 1986.

[32] K. Kondo, Geometry of elastic deformation and incompatibility, Memoirs of the Unifying Study of the Basic Problems in Engineering Science by Means of Geometry (K. Kondo, ed.), vol. 1, Division C, Gakujutsu Bunken Fukyo-Kai, 1955, pp. 5-17 (361-373). MF0080956 (18:332d)

[33] _ Non-Riemannien geometry of imperfect crystals from a macroscopic viewpoint, Memoirs of the Unifying Study of the Basic Problems in Engineering Science by Means of Geometry (K. Kondo, 
ed.), vol. 1, Division D-I, Gakujutsu Bunken Fukyo-Kai, 1955, pp. 6-17 (458-469). MR0080956 $(18: 332 \mathrm{~d})$

[34] A. Krawietz, Materialtheorie, Springer, Berlin, 1986. MR0959732 (90e:73001)

[35] E. Kröner, Kontinuumstheorie der Versetzungen und Eigenspannungen, Ergebnisse der Angewandten Mathematik, vol. 5, Springer, Berlin, 1958. MR0095615(20:2117)

[36] _ Dislocation: a new concept in the continuum theory of plasticity, J. Math. Phys. 42 (1962), $27-37$.

[37] E. Kröner and A. Seeger, Nichtlineare Elastizitätstheorie der Versetzungen und Eigenspannungen, Arch. Rat. Mech. Anal. 3 (1959), 97-119. MR0106587 (21:5318)

[38] E.H. Lee, Elastic-plastic deformation at finite strain, J. Appl. Mech. 36 (1969), 1-6.

[39] J. Lemaitre and J.L. Chaboche, Mécanique des matériaux solides, Dunod, Paris, 1985.

[40] M. Lucchesi and P. Podio-Guidugli, Materials with elastic range: a theory with a view toward applications. Part I, Arch. Rat. Mech. Anal. 102 (1988), 23-43. MR0938382 (90f:73008)

[41] J. Mandel, Equations constitutives et directeurs dans les milieux plastiques et viscoplastiques, Int. J. Solids Structures 9 (1973), 725-740.

[42] J.E. Marsden and J.R. Hughes, Mathematical Foundations of Elasticity, Prentice-Hall, Englewood Cliffs, New Jersey, 1983. MR1262126|(95h:73022)

[43] G. Maugin, Material Inhomogeneities in Elasticity, Applied Mathematics and Mathematical Computations, Chapman-Hall, London, 1993. MR,1250832 (95c:73001)

[44] _ The Thermomechanics of Nonlinear Irreversible Behaviors, Nonlinear Science, no. 27, World Scientific, Singapore, 1999.

[45] G.A. Maugin and M. Epstein, Geometrical material structure of elastoplasticity, Int. J. Plasticity 14 (1998), 109-115.

[46] A. Mayers, P. Schiebe, and O.T. Bruhns, Some comments on objective rates of symmetric eulerian tensors with application to eulerian strain rates, Acta Mech. 139 (2000), 91-103.

[47] C. Miehe, A theory of large-strain isotropic thermoplasticity based on metric transformation tensors, Archive Appl. Mech. 66 (1995), 45-64.

[48] A. Mielke, Finite elastoplasticity, Lie groups and geodesics on $S L(d)$, Sonderforschungsbereich 404 Bericht 2000/26, University of Stuttgart, 2000.

[49] A.I. Murdoch, Objectivity in classical continuum physics: a rationale for discarding the 'principle of invariance under superposed rigid body motions' in favour of purely objective considerations, Cont. Mech. Thermo. 15 (2003), 309-320. MR1986708 (2004f:74001)

[50] W. Muschik and L. Restuccia, Changing the observer and moving materials in continuum physics: objectivity and frame-indifference, Technische Mechanik 22 (2002), 152-160.

[51] P.M. Naghdi, A critical review of the state of finite plasticity, J. Appl. Math. Phys.(ZAMP) 41 (1990), 315-394. MR1058818

[52] A. Needleman, Material rate dependence and mesh sensitivity in localization problems, Comp. Meth. Appl. Mech. Engrg. 67 (1988), 69-85.

[53] P. Neff, Formulation of visco-plastic approximations in finite plasticity for a model of small elastic strains, Part I: Modelling, Preprint 2127, TU Darmstadt, 2000.

[54] — Mathematische Analyse multiplikativer Viskoplastizität, Ph.D. Thesis, TU Darmstadt, Shaker Verlag, ISBN:3-8265-7560-1, Aachen, 2000.

[55] - Formulation of visco-plastic approximations in finite plasticity for a model of small elastic strains, Part IIa: Local existence and uniqueness results, Preprint 2138, TU Darmstadt, 2001.

[56] _ On Korn's first inequality with nonconstant coefficients, Proc. Roy. Soc. Edinb. 132A (2002), 221-243. MR1884478 (2002k:74007)

[57] _ Finite multiplicative plasticity for small elastic strains with linear balance equations and grain boundary relaxation, Cont. Mech. Thermodynamics 15(2) (2003), no. DOI 10.1007/s00161002-0190-x, 161-195. MR.1970291 (2004f:74018)

[58] P. Neff and C. Wieners, Comparison of models for finite plasticity. A numerical study, Comput. Visual. Sci. 6 (2003), 23-35. MR.1985199 (2004d:74010)

[59] M. Ortiz and E.A. Repetto, Nonconvex energy minimization and dislocation structures in ductile single crystals, manuscript, 1993.

[60] _ Nonconvex energy minimization and dislocation structures in ductile single crystals, J. Mech. Phys. Solids 47 (1999), 397-462. MR1674064 (99k:73068)

[61] M. Ortiz, E.A. Repetto, and L. Stainier, A theory of subgrain dislocation structures, J. Mech. Phys. Solids 48 (2000), 2077-2114. MR.1778727 (2001d:74014) 
[62] M. Ortiz and L. Stainier, The variational formulation of viscoplastic constitutive updates, Comp. Meth. Appl. Mech. Engrg. 171 (1999), 419-444. MR,1685716 (99m:73036)

[63] W. Pompe, Korn's first inequality with variable coefficients and its generalizations, Comment. Math. Univ. Carolinae 44,1 (2003), 57-70. MF 2045845

[64] A. Raoult, Non-polyconvexity of the stored energy function of a St.Venant-Kirchhoff material, Aplikace Matematiky 6 (1986), 417-419. MF0870478 (88f:73028)

[65] M.B. Rubin, Plasticity theory formulated in terms of physically based microstructrural variablesPart I. Theory, Int. J. Solids Structures 31 (1994), no. 19, 2615-2634.

[66] C. Sansour and F.G. Kollmann, On theory and numerics of large viscoplastic deformation, Comp. Meth. Appl. Mech. Engrg. 146 (1996), 351-369.

[67] M. Silhavy, On transformation laws for plastic deformations of materials with elastic range, Arch. Rat. Mech. Anal. 63 (1976), 169-182. MR0669403 (58:32293)

[68] J.C. Simo, Numerical analysis and simulation of plasticity, Handbook of Numerical Analysis (P.G. Ciarlet and J.L. Lions, eds.), vol. VI, Elsevier, Amsterdam, 1998. MR1665428 (99m:73064)

[69] J.C. Simo and J.R. Hughes, Computational Inelasticity, Interdisciplinary Applied Mathematics, vol. 7, Springer, Berlin, 1998. MR,1642789 (99i:73038)

[70] J.C. Simo and M. Ortiz, A unified approach to finite deformation elastoplastic analysis based on the use of hyperelastic constitutive equations, Comp. Meth. Appl. Mech. Engrg. 49 (1985), 221-245.

[71] P. Steinmann, Views on multiplicative elastoplasticity and the continuum theory of dislocations, Int. J. Engrg. Sci. 34 (1996), 1717-1735.

[72] B. Svendsen and A. Bertram, On frame-indifference and form-invariance in constitutive theory, Acta Mechanica 132 (1997), 195-207. MR,1659588(99k:73008)

[73] T. Valent, Boundary Value Problems of Finite Elasticity, Springer, Berlin, 1988. MR0917733 (89c:73001)

[74] C. Wieners, M. Amman, S. Diebels, and W. Ehlers, Parallel 3-d simulations for porous media models in soil mechanics, Comp. Mech. 29 (2002), 75-87. 